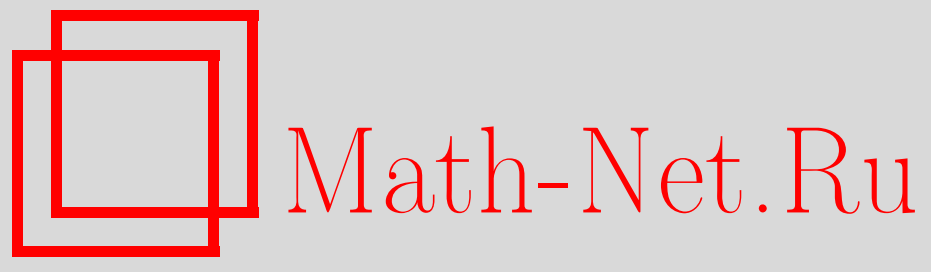

А. А. Боричев, Турбулентность в обобщенном уравнении Бюргерса, УМH, 2014, том 69, выпуск 6, 3-44

DOI: https://doi.org/10.4213/rm9629

Использование Общероссийского математического портала Math-Net.Ru подразумевает, что вы прочитали и согласны с пользовательским соглашением http://www . mathnet.ru/rus/agreement

Параметры загрузки:

IP: 54.92 .164 .108

26 апреля 2023 г., 07:19:36

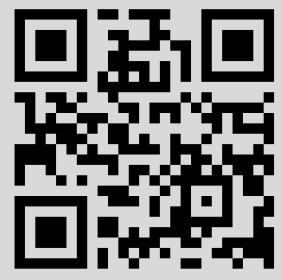




\section{Турбулентность в обобщенном уравнении Бюргерса}

\section{А. А. Боричев}

В работе дается обзор строгих результатов о турбулентности в обобщенном пространственно-периодическом уравнении Бюргерса:

$$
u_{t}+f^{\prime}(u) u_{x}=\nu u_{x x}+\eta, \quad x \in S^{1}=\mathbb{R} / \mathbb{Z},
$$

полученных в работах А.Э. Бирюка и автора [7], [9], [11], [12]. Здесь $f-$ гладкая строго выпуклая функция, а константа $0<\nu \ll 1$ соответствует коэффициенту вязкости.

Рассматривается случай отсутствия возмущения $(\eta=0)$ и случай, когда возмущение $\eta$ является случайным, гладким по $x$ и нерегулярным (возмущение толчками или белым шумом) по $t$. В обоих случаях получены точные оценки соболевских норм решения $u$, усредненных по времени и вероятности, имеющие вид $C \nu^{-\delta}, \delta \geqslant 0$, с одним и тем же значением $\delta$ в оценках сверху и снизу. Эти результаты дают точные оценки для мелкомасштабных величин, характеризующих турбулентность, что подтверждает физические предсказания из [6].

Библиография: 56 названий.

Ключевые слова: уравнение Бюргерса, стохастические уравнения в частных производных, турбулентность, перемежаемость, стационарная мера.

DOI: $10.4213 / \mathrm{rm} 9629$

\section{СОДЕРЖАНИЕ}

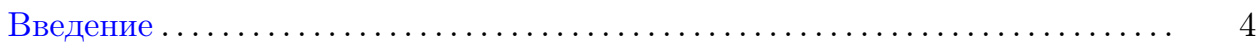

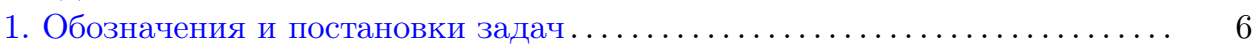

1.1. Функциональные пространства и соболевские нормы......... 6

1.2. Корректность и различные типы возмущений ............. 8

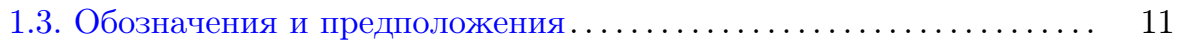

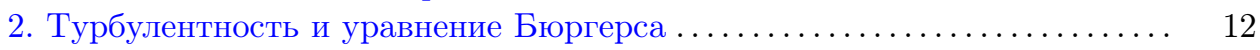

2.1. Турбулентность, теория K41, перемежаемость............. 12

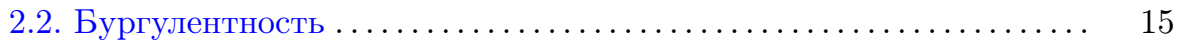

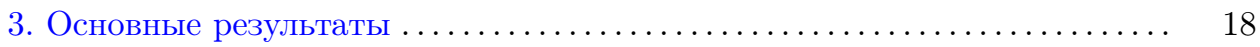

4. Оценки в невозмущенном случае .......................... 19

4.1. Оценки соболевских норм ......................... 20

4.2. Оценки для мелкомасштабных величин................ 25

(C) A. А. Боричев, 2014 
5. Оценки в случае случайного возмущения ................... 34

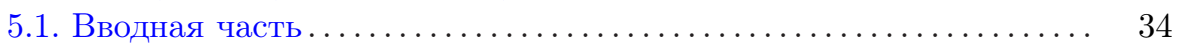

5.2. Оценки соболевских норм . . . . . . . . . . . . . . . . . . . . 34

5.3. Оценки мелкомасштабных величин ................... 37

6. Стационарная мера и сопутствующие вопросы ................ 38

Список литературы . . . . . . . . . . . . . . . . . . . . . . . . .

\section{Введение}

Обобщенное одномерное пространственно-периодическое уравнение Бюргерса

$$
\frac{\partial u}{\partial t}+f^{\prime}(u) \frac{\partial u}{\partial x}-\nu \frac{\partial^{2} u}{\partial x^{2}}=0, \quad \nu>0, \quad x \in S^{1}=\mathbb{R} / \mathbb{Z}
$$

(классическое уравнение Бюргерса [14] соответствует случаю $f(u)=u^{2} / 2$ ) представляет собой популярную модель уравнения Навье-Стокса. Действительно, оба эти уравнения имеют сходные нелинейный и диссипативный члены. Как следствие, физические аргументы, объясняющие различные теории гидродинамической турбулентности, обычно применимы для описания поведения решений уравнения Бюргерса. Соответственно, данное уравнение часто используется как модель для отработки теорий турбулентности и численных методов в теории турбулентных потоков. По поводу более подробной информации мы отсылаем читателя к работе [6].

При $\nu \ll 1$ и строго выпуклой $f$, т. е. такой, что

$$
f^{\prime \prime}(x) \geqslant \sigma>0, \quad x \in \mathbb{R},
$$

решения уравнения (1) обладают турбулентноподобным поведением, называемым турбулентностью Бюргерса или бургулентностью [27], [6]. Для упрощения изложения мы ограничимся решениями с нулевым средним по пространству:

$$
\int_{S^{1}} u(t, x) d x=0 \quad \forall t \geqslant 0 .
$$

Отметим, что это среднее не меняется со временем. Действительно, поскольку $и$ является 1-периодическим в пространстве, то

$$
\frac{d}{d t} \int_{S^{1}} u(t, x) d x=-\int_{S^{1}} f^{\prime}(u(t, x)) u_{x}(t, x) d x+\nu \int_{S^{1}} u_{x x}(t, x) d x=0 .
$$

Если среднее начального условия $u_{0}$ на $S^{1}$ есть $b \neq 0$, то мы можем рассмотреть функцию с нулевым средним

$$
v(t, x)=u(t, x+b t)-b,
$$

которая является решением уравнения (1), где вместо $f(y)$ мы берем $g(y)=$ $f(y+b)-b y$. Таким образом, предположение (3) не приводит к потере общности.

В данном обзоре мы рассматриваем уравнение (1) без внешнего возмущения и обобщенное уравнение Бюргерса с аддитивным возмущением, гладким 
в пространстве и нерегулярным по времени (см. п. 1.2). Мы приводим оценки, полученные А.Э. Бирюком и автором [7], [9], [11], [12] для соболевских норм, а также для масштаба длины диссипации и мелкомасштабных величин, важных в теории гидродинамической турбулентности - именно, для структурных функций и энергетического спектра. Данный обзор частично восходит к кандидатской $(\mathrm{PhD})$ диссертации автора [10], в которой некоторые технические моменты освещены более подробно.

Основное различие между уравнением Бюргерса в отсутствие возмущения и обобщенным уравнением Бюргерса с возмущением белым шумом проявляется в энергетической картине. В первом случае мы имеем диссипативную систему: $L_{2}$-норма решения убывает со временем. Как следствие, режим, в котором $\int_{S^{1}} u^{2} / 2$ диссипирует достаточно быстро (что дает усредненную по времени оценку для соболевских норм), является переходным. Зависимость этого режима от начального условия описывается определенной величиной $D$ (см. формулу (15) ниже). Наоборот, во втором случае, по прошествии времени, необходимого или на потерю энергии (если $u_{0}$ достаточно большое) или на подачу энергии (если $u_{0}$ мало), мы попадаем в квазистационарныци режим в том смысле, что в среднем, на больших временных интервалах, имеется приблизительное равновесие между скоростью диссипации $-\nu \mathrm{E}\|u\|_{1}^{2}$ и постоянной скоростью подвода энергии $I_{0}$.

В случае невозмущенного уравнения Бюргерса некоторые оценки сверху для мелкомасштабных величин хорошо известны. К примеру, лемма 4.1 настоящей работы является аналогом в периодическом случае односторонней липшицевой оценки, полученной О.А. Олейник, так что оценка сверху для структурной функции $S_{1}(\ell)$ вытекает из оценки решения в классе $B V$ функций ограниченной вариации. По поводу ссылок на классические аспекты теории скалярных законов сохранения мы отсылаем читателя к [19], [45], [49]. Относительно оценок сверху мелкомасштабных величин см. [38], [53]. Насколько нам известно, до работ А.Э. Бирюка и автора строгие оценки снизу отсутствовали.

Изучение мелкомасштабного поведения решений обобщенного (с возмущением) уравнения Бюргерса мотивировано задачами турбулентности. Побудительной причиной указанных исследований послужили пионерские работы С. Б. Куксина, который получил оценки сверху и снизу для соболевских норм через отрицательные степени вязкости для широкого класса уравнений (см. [41], [42] и ссылки в [42]). Более подробно результаты, полученные С. Б. Куксиным, А. Р. Ширикяном и другими для двумерного уравнения Навье-Стокса, описаны в монографии [43] и приведенных там источниках.

Представленные в настоящей работе оценки соболевских норм и мелкомасштабных величин являются асимптотически точными в том смысле, что вязкость входит в оценки снизу и сверху в одинаковых отрицательных степенях. Такие оценки не получены для более сложных уравнений, рассмотренных в [41]-[43].

В данном обзоре мы также останавливаемся на проблеме существования и единственности инвариантной меры для стохастического обобщенного уравнения Бюргерса. В случае нелинейности $u u_{x}$ данный вопрос был исследован 
Я. Г. Синаем [51], [52], случай невязкого предела $\nu \rightarrow 0$ рассматривался В. И, К. М. Ханиным, А. Е. Мазелем и Я. Г. Синаем [21] (см. также [28], [31]). Здесь, следуя нашей работе [12], мы представляем достаточно простой подход к данной задаче, основанный на использовании $L_{1}$-сжимаемости потока, соответствующего уравнению, а также метода каплинга.

Мы не рассматриваем другие аспекты бургулентности, такие как невязкий предел, поведение решений для силы, не гладкой в пространстве, и некомпактный случай. Здесь мы отсылаем читателя к обзору Ж. Бека и К. М. Ханина [6], в котором рассмотрены физические аспекты теории бургулентности, а также к обзору Ю. Ю. Бахтина [3], в котором обсуждаются смежные вероятностные и эргодические результаты.

Структура работы. В разделе 1 мы вводим используемые обозначения и описываем постановку задачи. В разделе 2 мы напоминаем положения теории К41 и приводим физические предсказания бургулентности. Основные результаты формулируются в разделе 3 . В разделе 4 рассматривается решение $u(t, x)$ невозмущенного уравнения (1). Далее, в п. 4.1, приводится оценка сверху для величины

$$
\max _{s \in[t, t+1], x \in S^{1}} u_{x}(s, x), \quad t \geqslant 1 .
$$

С использованием этой оценки мы получаем оценки сверху и снизу для соболевских норм решения $u$. Приложения полученных результатов к теории бургулентности даются в п. 4.2. Именно, мы приводим точные оценки сверху и снизу для масштаба длины диссипации, приращений и спектральной асимптотики потока $u(t, x)$, которые имеют место равномерно по $\nu \leqslant \nu_{0}$. Величина $\nu_{0}>0$ зависит только от $f$ и от начального условия. Эти результаты дают строгое обоснование физических предсказаний для мелкомасштабных величин, характеризующих бургулентность.

В последних двух разделах статьи мы рассматриваем обобщенное уравнение Бюргерса со случайным возмущением. В разделе 5 мы получаем аналоги результатов из раздела 4 , также подтверждающие соответствующие физические предсказания [6]. Вопросы существования и единственности стационарной меры рассматриваются в разделе 6 .

\section{1. Обозначения и постановки задач}

Все рассматриваемые в статье функции предполагаются действительнозначными, за исключением раздела 2 , где для векторов из $\mathbb{R}^{3}$ используется полужирный шрифт.

1.1. Функциональные пространства и соболевские нормы. Рассмотрим интегрируемую функцию $v$ с нулевым средним на $S^{1}$. Для $p \in[1, \infty]$ через $|v|_{p}$ мы обозначим ее $L_{p}$-норму $\left(L_{2}\right.$-норма обозначается через $\left.|v|\right)$. Скалярное произведение в $L_{2}$ обозначается через $\langle\cdot, \cdot\rangle$. На протяжении работы $L_{p}$, $p \in[1, \infty]$, обозначает пространство функций из $L_{p}\left(S^{1}\right)$ с нулевым средним. Аналогично, $C^{\infty}$ есть пространство $C^{\infty}$-гладких функций на $S^{1}$ с нулевым средним. 
Для неотрицательного целого $m$ и $p \in[1, \infty]$ через $W^{m, p}$ обозначим пространство Соболева функций $v$ на $S^{1}$ с нулевым средним и конечной однородной нормой

$$
|v|_{m, p}=\left|\frac{d^{m} v}{d x^{m}}\right|_{p}
$$

В частности, $W^{0, p}=L_{p}$ при $p \in[1, \infty]$. В случае $p=2$ мы пишем $H^{m}$ вместо $W^{m, 2}$, при этом соответствующая норма сокращенно записывается как $\|v\|_{m}$.

Поскольку длина $S^{1}$ равна 1 , мы имеем

$$
|v|_{1} \leqslant|v|_{\infty} \leqslant|v|_{1,1} \leqslant|v|_{1, \infty} \leqslant \cdots \leqslant|v|_{m, 1} \leqslant|v|_{m, \infty} \leqslant \cdots .
$$

Напомним следующий вариант классического неравенства Гальярдо-Ниренберга (см. [20; приложение]).

Лемма 1.2. Пусть $v$ - гладкая функиия с нулевым средним на $S^{1}$. Тогда

$$
|v|_{\beta, r} \leqslant C|v|_{m, p}^{\theta}|v|_{q}^{1-\theta}
$$

где $m>\beta \geqslant 0$, a $r$ определяется из соотношения

$$
\frac{1}{r}=\beta-\theta\left(m-\frac{1}{p}\right)+(1-\theta) \frac{1}{q}
$$

при условии, что $\theta=\beta / m$ при $p=1$ или $p=\infty ; \beta / m \leqslant \theta<1$ в противном случае. Константа $C$ зависит от $m, p, q, \beta, \theta$.

Всюду в работе такое неравенство будет называться неравенством Гальярдо-Ниренберга.

Для $s \geqslant 0$ через $H^{s}$ мы обозначим пространство Соболева функций $v$ с нулевым средним на $S^{1}$ и конечной нормой

$$
\|v\|_{s}=(2 \pi)^{s}\left(\sum_{k \in \mathbb{Z}}|k|^{2 s}|\widehat{v}(k)|^{2}\right)^{1 / 2},
$$

где $\widehat{v}(k)$ - комплексные коэффициенты Фурье функции $v(x)$. Для целых $s=m$ такая норма совпадает с введенной выше $H^{m}$-нормой. При $s \in(0,1)$ норма $\|v\|_{s}$ эквивалентна норме

$$
\|v\|_{s}^{\prime}=\left(\int_{S^{1}}\left(\int_{0}^{1} \frac{|v(x+\ell)-v(x)|^{2}}{\ell^{2 s+1}} d \ell\right) d x\right)^{1 / 2}
$$

(см. [1], [54]).

Как обычно, индексы $t$ и $x$, возможно повторяющиеся, обозначают взятие частной производной по соответствующей переменной. Через $v^{(m)}$ обозначается производная порядка $m$ от функции $v$ по переменной $x$. Для краткости мы записываем $v(t, \cdot)$ как $v(t)$. 
1.2. Корректность и различные типы возмущений. В разделе 4 мы рассматриваем невозмущенное уравнение (1) с $C^{\infty}$-гладким начальным условием $u_{0}$. Это уравнение имеет единственное решение в классе $C^{\infty}$ (см., к примеру, [39; гл. 5]).

В разделе 5 изучается обобщенное уравнение Бюргерса с двумя различными типами аддитивного возмущения в правой части. При этом, как и выше, предполагается $C^{\infty}$-гладкость начального условия $u_{0}$. Так как возмущение всегда имеет нулевое среднее в пространстве, а начальное условие удовлетворяет условию (3), то все решения удовлетворяют (3) для любого момента времени.

Мы начинаем со случая возмущения толчками. Для этой цели мы наделяем пространство $L_{2} \sigma$-алгеброй $(\Omega, \mathscr{F})$ борелевских подмножеств и на вероятностном пространстве $(\Omega, \mathscr{F}, \mathrm{P})$ рассматриваем случайную величину $\zeta=\zeta^{\omega}$ со значениями в $L_{2}$. Мы предполагаем, что $\zeta$ удовлетворяет следующим трем условиям.

(i) Нетривиальность:

$$
\mathrm{P}(\zeta \equiv 0)<1 .
$$

(ii) Конечность моментов для соболевских норм: для каждого $m \geqslant 0$

$$
I_{m}=\mathrm{E}\|\zeta\|_{m}^{2}<+\infty .
$$

(iii) Равенство нулю математического ожидания:

$$
\mathrm{E} \zeta \equiv 0
$$

Несложно построить в явном виде случайную величину $\zeta$, удовлетворяющую условиям (i)-(iii). K примеру, предположим, что действительные коэффициенты Фурье случайной величины $\zeta$, определенные при $k>0$ соотношениями

$$
\begin{aligned}
& a_{k}(\zeta)=\sqrt{2} \int_{S^{1}} \cos (2 \pi k x) u(x) d x \\
& b_{k}(\zeta)=\sqrt{2} \int_{S^{1}} \sin (2 \pi k x) u(x) d x
\end{aligned}
$$

являются независимыми случайными величинами с нулевым средним и экспоненциальными моментами, достаточно быстро стремящимися к 1 при $k \rightarrow+\infty$.

Теперь пусть $\zeta_{i}=\zeta_{i}^{\omega}, i \in \mathbb{N},-$ независимые одинаково распределенные случайные величины с тем же распределением, что и $\zeta$. Последовательность $\left(\zeta_{i}\right)_{i \geqslant 1}$ является случайной величиной, определенной на вероятностном пространстве, являющемся счетным произведением копий $\Omega$. Здесь и ниже мы будем обозначать такое пространство через $\Omega$, при этом смысл обозначений $\mathscr{F}$ и $\mathrm{P}$ изменяется соответственно.

Для $\omega \in \Omega$ сила возмущения толчками $\xi^{\omega}$ по определению является распределением

$$
\xi^{\omega}(t, x)=\sum_{i=1}^{+\infty} \delta_{t=i} \zeta_{i}^{\omega}(x),
$$

где $\delta_{t=i}-$ мера Дирака в момент времени $i$. 
Уравнение с возмущением толчками соответствует случаю, когда в правой части (1) вместо 0 рассматривается сила возмущения толчками:

$$
\frac{\partial u}{\partial t}+f^{\prime}(u) \frac{\partial u}{\partial x}-\nu \frac{\partial^{2} u}{\partial x^{2}}=\xi^{\omega} .
$$

Это означает, что при целых $i \geqslant 1$ в моменты времени $i$ к решению $u(x)$ мы прибавляем величину толчка $\zeta_{i}^{\omega}(x)$, а между целыми моментами времени решение $u$ удовлетворяет уравнению (1). Мы сделаем дополнительное предположение, что решение является непрерывной справа функцией времени.

Существование и единственность пространственно-гладких решений уравнения (7) непосредственно следует из соответствующего факта для невозмущенного уравнения.

Другим типом рассматриваемого возмущения является возмущение белым шумом. Эвристически такое возмущение соответствует масштабированному пределу сил возмущения все более и более частыми толчками.

С каждым пространством $W^{m, p}$ мы ассоциируем $\sigma$-алгебру борелевских множеств. Рассмотрим винеровский процесс со значениями в $L_{2}$

$$
w(t)=w^{\omega}(t), \quad \omega \in \Omega, \quad t \geqslant 0,
$$

определенный на полном вероятностном пространстве $(\Omega, \mathscr{F}, \mathrm{P})$, а также согласованную фильтрацию $\left\{\mathscr{F}_{t}, t \geqslant 0\right\}$ (а именно, такую фильтрацию, что $w(t)$ $\mathscr{F}_{t}$-измерима при $t \geqslant 0$, при этом $\mathscr{F} t$ и $\sigma$-алгебра, порожденная случайными величинами $w(t+s)-w(t), s \geqslant 0$, являются независимыми).

Мы предполагаем, что $w(t) \in H^{m}$ почти наверное при любых $m$ и $t \geqslant 0$. Таким образом, при $\zeta, \chi \in L_{2}$

$$
\mathrm{E}(\langle w(s), \zeta\rangle\langle w(t), \chi\rangle)=\min (s, t)\langle Q \zeta, \chi\rangle,
$$

где $Q$ - симметричный оператор, определяющий непрерывное отображение $Q: L_{2} \rightarrow H^{m}$ для каждого $m$. Итак, $w(t) \in C^{\infty}$ для каждого $t$ почти наверное. Начиная с этого места мы переопределим винеровский процесс таким образом, чтобы данное свойство было выполнено при всех $\omega \in \Omega$. Для краткости вместо $w(t)(x)$ мы пишем $w(t, x)$. Для $m \geqslant 0$ через $I_{m}$ обозначим величину

$$
I_{m}=\operatorname{Tr}_{H^{m}}(Q)=\mathrm{E}\|w(1)\|_{m}^{2} .
$$

Более подробно о винеровских процессах в гильбертовых пространствах см. $[17 ;$ гл. 4] и [44].

Явное построение $w(t)$ не вызывает труда. К примеру, мы можем рассмотреть частный случай диагонального шума

$$
w(t)=\sqrt{2} \sum_{k \geqslant 1} a_{k} w_{k}(t) \cos (2 \pi k x)+\sqrt{2} \sum_{k \geqslant 1} b_{k} \widetilde{w}_{k}(t) \sin (2 \pi k x),
$$

где $w_{k}(t), \widetilde{w}_{k}(t), k>0,-$ стандартные независимые винеровские процессы, причем

$$
I_{m}=\sum_{k \geqslant 1}\left(a_{k}^{2}+b_{k}^{2}\right)(2 \pi k)^{2 m}<\infty
$$


при каждом $m$. Всюду ниже $d w(s)$ будет обозначать стохастический дифференциал, соответствующий винеровскому процессу $w(s)$ в пространстве $L_{2}$.

Теперь предположим, что $m \geqslant 0$ фиксировано. По теореме Ферника [44; теорема 3.3.1] существуют $\lambda_{m}, C_{m}>0$ такие, что

$$
\mathrm{E} \exp \left(\frac{\lambda_{m}\|w(T)\|_{m}^{2}}{T}\right) \leqslant C_{m}, \quad T \geqslant 0
$$

Отсюда по максимальному неравенству Дуба для бесконечномерных субмартингалов [17; теорема 3.8, (ii)] получаем

$$
\mathrm{E} \sup _{t \in[0, T]}\|w(t)\|_{m}^{p} \leqslant\left(\frac{p}{p-1}\right)^{p} \mathrm{E}\|w(t)\|_{m}^{p}<+\infty
$$

при любых $T>0$ и $p \in(1, \infty)$.

Уравнение с возмущением слабым шумом получается при замене 0 слабой производной $\eta^{\omega}=\partial w^{\omega} / \partial t$ в правой части уравнения (1). Здесь $w^{\omega}(t), t \geqslant 0,-$ определенный выше винеровский процесс.

ОПРЕДЕЛЕНИЕ 1.3. Пусть $T \geqslant 0$. Мы говорим, что процесс $u(t, x)=u^{\omega}(t, x)$ со значениями в $H^{1}$ является решением уравнения

$$
\frac{\partial u^{\omega}}{\partial t}+f^{\prime}\left(u^{\omega}\right) \frac{\partial u^{\omega}}{\partial x}-\nu \frac{\partial^{2} u^{\omega}}{\partial x^{2}}=\eta^{\omega}
$$

при $t \geqslant T$, если:

(i) при любом $t \geqslant T$ отображение $\omega \mapsto u^{\omega}(t, \cdot)$ является $\mathscr{F}_{t}$-измеримым;

(ii) для любого $\omega$ отображение $t \mapsto u^{\omega}(t, \cdot)$ непрерывно в $H^{1}$ при $t \geqslant T$, при этом

$$
u^{\omega}(t)=u^{\omega}(T)-\int_{T}^{t}\left(\nu L u^{\omega}(s)+\frac{1}{2} B\left(u^{\omega}\right)(s)\right) d s+w^{\omega}(t)-w^{\omega}(T),
$$

где

$$
B(u)=2 f^{\prime}(u) u_{x}, \quad L=-\partial_{x x} .
$$

Для краткости решения при $t \geqslant 0$ мы будем называть просто решениями.

Существование и единственность гладких решений уравнения (10) доказывается с использованием аппарата слабых (mild) решений (см. [18; гл. 14]). Поскольку возмущение и начальное условие являются гладкими по $x$, то отображение $t \mapsto u(t)$ непрерывно в пространстве $H^{m}$ для каждого $m$ и отображение $t \mapsto u(t)-w(t)$ имеет $C^{\infty}$-гладкую производную по $x$ при всех $t$ почти наверное.

Для решения $u(t, x)$ уравнения (10) рассмотрим функционал $G_{m}(u(t))=$ $\|u(t)\|_{m}^{2}$ и воспользуемся формулой Ито [17; теорема 4.17]. Имеем

$$
\begin{gathered}
\|u(t)\|_{m}^{2}=\left\|u_{0}\right\|_{m}^{2}-\int_{0}^{t}\left(2 \nu\|u(s)\|_{m+1}^{2}+\left\langle L^{m} u(s), B(u)(s)\right\rangle\right) d s+t I_{m} \\
+2 \int_{0}^{t}\left\langle L^{m} u(s), d w(s)\right\rangle
\end{gathered}
$$


(напомним, что $\left.I_{m}=\operatorname{Tr}\left(Q_{m}\right)\right)$. Следовательно, имеет место соотношение

$$
\frac{d}{d t} \mathrm{E}\|u(t)\|_{m}^{2}=-2 \nu \mathrm{E}\|u(t)\|_{m+1}^{2}-\mathrm{E}\left\langle L^{m} u(t), B(u)(t)\right\rangle+I_{m}
$$

Так как $\langle u, B(u)\rangle=0$, то при $m=0$ это соотношение принимает вид

$$
\frac{d}{d t} \mathrm{E}|u(t)|^{2}=I_{0}-2 \nu \mathrm{E}\|u(t)\|_{1}^{2} .
$$

1.3. Обозначения и предположения. При рассмотрении пространства $W^{m, p}$ с соболевской нормой мы считаем, что $\gamma=\gamma(m, p):=\max (0, m-1 / p)$.

В п. 2.1 через $\mathbf{v}(t, \mathbf{x})$ мы обозначаем скорость трехмерного потока с периодом 1 по каждой из пространственных координат. Всюду далее $u(t, x)$ обозначает решение обобщенного уравнения Бюргерса с заданным начальным значением $u_{0}=u(0, \cdot)$. В разделе 4 мы рассматриваем уравнение $(1)$ при предположениях (2), (3). В разделе 5 исследуется уравнение (10) при предположениях (2), (3) и дополнительном условии

$$
\forall m \geqslant 0, \quad \exists h \geqslant 0, C_{m}>0: \quad\left|f^{(m)}(x)\right| \leqslant C_{m}(1+|x|)^{h}, \quad x \in \mathbb{R},
$$

где $h=h(m)$ - функция, для которой

$$
1 \leqslant h(1)<2
$$

(оценка снизу для $h(1)$ вытекает из (2)). Результаты раздела 5 также имеют место для уравнения с возмущением толчками (7) при тех же предположениях, что и для уравнения (10) (за исключением условия (14), которое не нужно).

При рассмотрении обобщенного уравнения Бюргерса со случайным возмущением через $\mathrm{P}$ и $\mathrm{E}$ будут обозначаться вероятностная мера на $\Omega$ и соответствующее математическое ожидание (см. п. 1.2).

Все величины, обозначаемые буквой $C$ (с верхними или нижними индексами), будут положительными и неслучайными, зависящими (если не оговорено противное) только от следующих параметров:

- статистических свойств возмущения - при анализе в рамках теории K41;

- функции $f$, определяющей нелинейность $f^{\prime}(u) u_{x}$, а также параметра

$$
D=\max \left(\left|u_{0}\right|_{1}^{-1},\left|u_{0}\right|_{1, \infty}\right)
$$

характеризующего общность начального условия - при изучении невозмущенного обобщенного уравнения Бюргерса;

- функции $f$, определяющей нелинейность $f^{\prime}(u) u_{x}$, а также статистических свойств возмущения - при изучении обобщенного уравнения Бюргерса со случайным возмущением; в случае возмущения толчками, говоря о статистических свойствах, мы имеем в виду функцию распределения независимых одинаково распределенных случайных величин $\zeta_{i}$, а в случае возмущения белым шумом корреляционный оператор $Q$ винеровского процесса $w$, определяющий случайное возмущение. 
В частности, эти параметры никогда не зависят от коэффициента вязкости $\nu$. Через $C\left(a_{1}, \ldots, a_{k}\right)$ мы будем обозначать константы, зависящие, в частности, от параметров $a_{1}, \ldots, a_{k}$. Запись $X \stackrel{a_{1}, \ldots, a_{k}}{\lesssim} Y$ означает, что $X \leqslant C\left(a_{1}, \ldots, a_{k}\right) Y$. Двойное неравенство

$$
Y \stackrel{a_{1}, \ldots, a_{k}}{\lesssim} X \stackrel{a_{1}, \ldots, a_{k}}{\lesssim} Y
$$

будет сокращенно записываться в виде $X \stackrel{a_{1}, \ldots, a_{k}}{\sim} Y$. В частности, записи $X \lesssim Y$ и $X \sim Y$ означают соответственно, что $X \leqslant C Y$ и $C^{-1} Y \leqslant X \leqslant C Y$. Отметим, что в такой записи никогда не используется параметр $\nu$. Иными словами, зависимость от вязкости всегда явно указывается.

Мы также полагаем $g^{-}=\max (-g, 0)$ и $g^{+}=\max (g, 0)$.

В п. 2.1 угловые скобки $\langle\cdot\rangle$ обозначают математическое ожидание. Значение скобок $\{\cdot\}$ в детерминированном случае определяется в п. 4.1 (где они означают усреднение по времени), а в случае случайного возмущения - в п. 5.3 (где они означают усреднение по времени и операцию взятия математического ожидания). Определения масштабов длин, а также мелкомасштабных величин (структурных функций $S_{p, \alpha}$ и $S_{p, 1}=S_{p}$ и энергетического спектра $E(k)$ ) зависят от ситуации - см. пп. 2.1, 2.2, 4.2 и 5.3 .

\section{2. Турбулентность и уравнение Бюргерса}

2.1. Турбулентность, теория K41, перемежаемость. Хорошо известно, что дать точное определение турбулентности весьма проблематично. Тем не менее имеется согласие насчет того, что некоторые свойства считаются характеристиками турбулентности: большое число степеней свободы, непредсказуемость/хаос, (мелкомасштабная) нерегулярность и т. п. Данный вопрос обсуждается в деталях в [26], [55]. Мы же ограничимся только представлением (в слегка модифицированной форме) словаря теории турбулентности, который имеет важное значение в изучении модели Бюргерса. В частности, мы считаем, что рассматриваемый поток $\mathbf{v}(t, \mathbf{x})$ является периодическим в пространстве, при этом мы не останавливаемся на физическом содержании теории K41 в данной постановке. Без ограничения общности можно предполагать, что поток $\mathbf{v}$ является 1-периодическим по каждой из координат $x_{1}, x_{2}, x_{3}$. Через $\nu$ обозначается коэффициент вязкости; мы будем рассматривать турбулентный режим, т. е. случай $0<\nu \ll 1$.

Мы определим пространственный масштаб как обратную величину к рассматриваемой частоте. В частности, коэффициенты Фурье $\widehat{\mathbf{v}}(\mathbf{k})$ при больших значениях $\mathbf{k}$ или, в физическом пространстве, приращения $\mathbf{v}(\mathbf{x}+\mathbf{r})-\mathbf{v}(\mathbf{x})$ при малых значениях $\mathbf{r}$ являются прототипами мелкомасштабных величин.

Начала современной теории турбулентности были заложены в трех работах А.Н. Колмогорова [33]-[35], опубликованных в 1941 г. Соответственно, мы будем ссылаться на содержание этих работ, используя термин “теория K41".

Философия теории K41 состоит в том, что, несмотря на то что характеристики турбулентного потока, очевидно, индивидуальны (будучи зависимыми от возмущения и/или начальных условий), мелкомасштабные характеристики проявляют ряд нетривиальных свойств универсального характера. Чтобы 
сделать более понятным это утверждение, нам потребуется ввести несколько определений.

Масштаб длинь диссипации $\ell_{d}$ есть наименьший масштаб, на котором при всех $|\mathbf{k}| \succeq \ell_{d}^{-1}$ коэффициенты Фурье функции $\mathbf{v}$ убывают суперполиномиально по $|\mathbf{k}|$ и равномерно по $\nu$. Интервал $\mathbf{J}_{\text {diss }}=\left(0, \ell_{d}\right]$ называется диссипационным интервалом. Согласно теории $\mathrm{K} 41, \ell_{d}=C \nu^{3 / 4}$. Энергетический интервал $\mathbf{J}_{\text {energ }}=\left(\ell_{e}, 1\right]$ состоит из всех таких масштабов, что на соответствующие моды Фурье приходится большая часть $L^{2}$-нормы функции v:

$$
\sum_{|\mathbf{k}|<\ell_{e}^{-1}}\left\langle|\widehat{\mathbf{v}}(\mathbf{k})|^{2}\right\rangle \gg \sum_{|\mathbf{k}| \geqslant \ell_{e}^{-1}}\left\langle|\widehat{\mathbf{v}}(\mathbf{k})|^{2}\right\rangle .
$$

Теория K41 утверждает, что $\ell_{e}=C$.

Наконец, $\mathbf{J}_{\text {inert }}=\left(\ell_{d}, \ell_{e}\right]$ - это инерционный интервал. Согласно теории $\mathrm{K} 41$, $\mathbf{J}_{\text {inert }}=\left(C \nu^{3 / 4}, C\right]$ (см. рис. 1). Этот интервал представляет наибольший интерес, поскольку на нем поток проявляет нетривиальное мелкомасштабное поведение, которое будет более подробно описано ниже. Эвристически в диссипационном интервале затухание соответствует исчезновению всех важных свойств потока, а в энергетическом интервале поведение потока существенно определяется крупномасштабными свойствами (т. е. случайным возмущением).

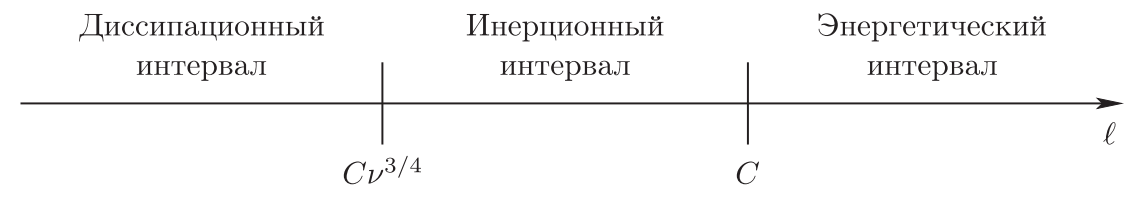

Рис. 1. Колмогоровские масштабы

Для описания мелкомасштабного поведения потока $\mathbf{v}(t, \mathbf{x})$ в фиксированныи момент времени используются две величины:

- продольная структурная функиия

$$
S_{p}^{\|}(\mathbf{x}, \mathbf{r})=\left\langle\left|\frac{(\mathbf{v}(\mathbf{x}+\mathbf{r})-\mathbf{v}(\mathbf{x})) \cdot \mathbf{r}}{|\mathbf{r}|}\right|^{p}\right\rangle ;
$$

- энергетический спектр

$$
E(k)=\frac{\sum_{|\mathbf{n}| \in\left[M^{-1} k, M k\right]}\left\langle|\widehat{\mathbf{v}}(\mathbf{n})|^{2}\right\rangle}{\sum_{|\mathbf{n}| \in\left[M^{-1} k, M k\right]} 1},
$$

т. е. усреднение $\left\langle|\widehat{\mathbf{v}}(\mathbf{n})|^{2}\right\rangle$ по слою $\mathbf{n}$, на котором $|\mathbf{n}| \sim k$.

Теория K41 предсказывает, что, при некоторых условиях на поток, для $\ell=$ $|\mathbf{r}| \in \mathbf{J}_{\text {inert }}$ и для каждого х имеет место соотношение

$$
S_{p}^{\|}(\mathbf{x}, \mathbf{r}) \stackrel{p}{\sim} \ell^{p / 3}, \quad p \geqslant 0
$$


С другой стороны, согласно теории K41 (см. [46], [47]) при $k^{-1} \in \mathbf{J}_{\text {inert }}$

$$
E(k) \sim k^{-5 / 3}
$$

Предсказания теории K41 находятся в хорошем соответствии с экспериментальными данными и данными численных расчетов для энергетического спектра и структурных функций $S_{p}$ с $p=2,3$. Однако между ними имеются важные различия, касающиеся функций $S_{p}$ с $p \geqslant 4$ [26; гл. 8]. Одно из возможных объяснений данных различий основывается на понятии пространственной перемежаемости.

Мы говорим, что функция является перемежающейся в пространстве, если в фиксированный момент времени она очень сильно возбуждена на малом подмножестве своей области определения (как, например, функция, изображенная на рис. 2).

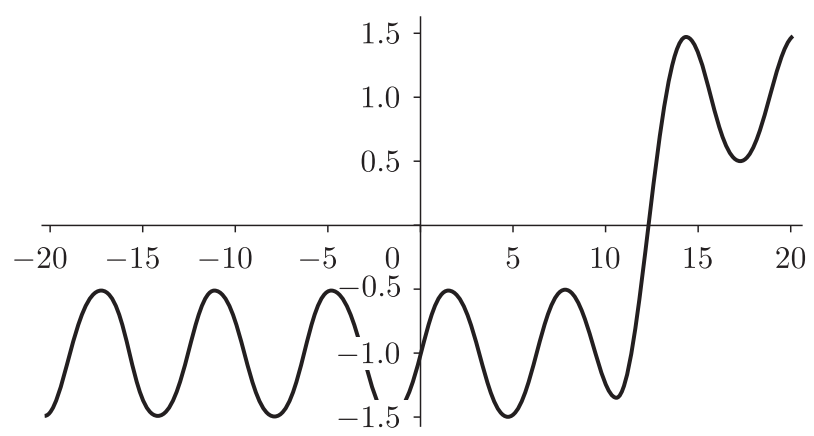

Рис. 2. Пример функции с мелкомасштабной перемежаемостью

Перемежаемость в масштабе $\ell$ численно определяется эксцессом, определяемым формулой

$$
F(\ell)=\frac{S_{4}^{\|}(\ell)}{S_{2}^{\|}(\ell)^{2}},
$$

- чем больше эксцесс, тем больше функция перемежается. Как следствие, теория K41 не может предсказать свойства перемежаемости, наблюдаемые на инерционном интервале в турбулентных потоках - к таким явлениям относится, например, растяжение вихревых нитей, где перемежаемость явно проявляется [50]. Действительно, при $\ell \in J_{2}$ теория K41 предсказывает, что

$$
F(\ell) \sim \frac{\ell^{4 / 3}}{\left(\ell^{2 / 3}\right)^{2}}=1 .
$$

Каждая из двух параллельных теорий, восходящих соответственно к самому А. Н. Колмогорову [36] и к У. Фришу и Дж. Паризи [48], объясняет расхождение между теорией K41 и экспериментальными и численными результатами, подчеркивая роль пространственной перемежаемости. 
2.2. Бургулентность. Одномерное уравнение Бюргерса

$$
u_{t}+u u_{x}=\nu u_{x x}
$$

где $\nu>0$ - коэффициент вязкости, было впервые рассмотрено А. Р. Форсайтом [24] и Г. Бейтменом [5] в первой половине XX века. Здесь мы ограничимся только рассмотрением пространственно-периодического случая, который, после изменения масштаба, сводится к случаю $\mathrm{x} \in S^{1}=\mathbb{R} / \mathbb{Z}$.

В 1950-х годах значительно возрос интерес к уравнению (20). Это уравнение, в частности, исследовалось голландским физиком Й. М. Бюргерсом, чье имя оно впоследствии получило ([13], [14]; см. также [4]). Целью Бюргерса было рассмотрение упрощенного варианта уравнения Навье-Стокса для несжимаемой жидкости

$$
\mathbf{u}_{t}+(\mathbf{u} \cdot \nabla) \mathbf{u}=\nu \Delta \mathbf{u}-\nabla p, \quad \nabla \cdot \mathbf{u}=0,
$$

с которым уравнение Бюргерса имеет много формальных аналогий. Дж. фон Нейман надеялся также, что это поможет понять поведение решений уравнения Навье-Стокса [56; с. 437].

При помощи преобразования Коула-Хопфа-Флорина ([16], [23], [30]; см. [8] по поводу исторических замечаний) уравнение Бюргерса сводится к уравнению теплопроводности. Действительно, если $u$ - решение уравнения $(20)$, соответствующее начальному условию $u_{0}$, то $u(t, x)$ есть производная в пространстве от функции

$$
-2 \nu \log (\phi(t, x))
$$

где $\phi$ - решение уравнения теплопроводности

$$
\phi_{t}=\nu \phi_{x x}
$$

соответствующее начальному условию $\phi_{0}=\exp \left(-H_{0} /(2 \nu)\right)$. Здесь $H_{0}-$ первообразная для $u_{0}$. Данное преобразование можно также применить к многомерному потенциальному уравнению Бюргерса:

$$
\mathbf{u}_{t}+(\mathbf{u} \cdot \nabla) \mathbf{u}=\nu \Delta \mathbf{u}, \quad \mathbf{u}=-\nabla \psi .
$$

Тот факт, что уравнение Бюргерса сводится к уравнению теплопроводности, означает, что оно интегрируемо и, как следствие, его решения не могут иметь хаотического поведения. Следует, однако, заметить, что преобразование Коула-Хопфа-Флорина включает в себя степень величины, разделенной на $\nu$, и не дает явной информации о мелкомасштабном поведении решений в режиме турбулентности, соответствующем значениям $0<\nu \ll 1$. Более того, данное преобразование не может быть применено к обобщенному уравнению Бюргерса, рассматриваемому в настоящему обзоре. По этой причине мы не обращаемся к преобразованию Коула-Хопфа-Флорина - наши аргументы распространяются на обобщенное уравнение Бюргерса (1) при дополнительном предположении (2).

Мелкомасштабное поведение решений уравнения Бюргерса интенсивно изучалось на качественном уровне в физической литературе [2], [15], [25], [32], [37]. Имеется согласие насчет поведения приращений и энергетического спектра 
в инерционном интервале $\mathbf{J}_{\text {inert }}=(C \nu, C]$. Для объяснения физических соображений из работы [2] нам потребуется иметь более детальное представление о структуре решений уравнения (20). Мы предположим, что начальное условие $u_{0}$ и его производная имеют амплитуду порядка 1.

Для начала рассмотрим невязкое уравнение Хопфа, соответствующее предельному случаю $\nu=0$ уравнения (20). Его решение, гладкое лишь на конечном временном интервале, может быть явно построено с использованием метода характеристик (см., например, [19]). Соответственно, если решение остается гладким, то значение $u$ является постоянным вдоль прямых $\left(t, x+t u_{0}(x)\right)$ в пространстве-времени. Однако, если $u_{0}$ не является постоянным, то линии, соответствующие различным значениям $u_{0}$, пересекаются по прошествии конечного времени, что препятствует существованию гладких решений. Тем не менее слабое энтропийное решение может быть однозначно определено для всех моментов времени в классе $B V\left(S^{1}\right)$ функций ограниченной вариации на $S^{1}$. Такое решение есть предел в $L_{1}$ классических решений вязкого уравнения при $\nu \rightarrow 0$. Более точно, данное решение имеет $N$-волновое поведение [22], т. е. состоит из волн, сходных с русской буквой "И" (см. рис. 3). Иными словами, при фиксированном (достаточно большом) значении времени $t$ решение $u(t, \cdot)$ последовательно меняется между отрицательными разрывами и областями гладкости, в которых производная положительна и сравнима с 1. Это есть очевидное проявление мелкомасштабной перемежаемости в пространстве. Отметим, что решения уравнения (20) остаются сравнимымы с 1 в течение времени, сравнимого с 1 . С другой стороны, при $t \rightarrow+\infty$ решения убывают со скоростью не менее $C t^{-1}$ равномерно по $\nu$ в любом из пространств Лебега $L_{p}, 1 \leqslant p \leqslant+\infty$ (см., например, [40]).

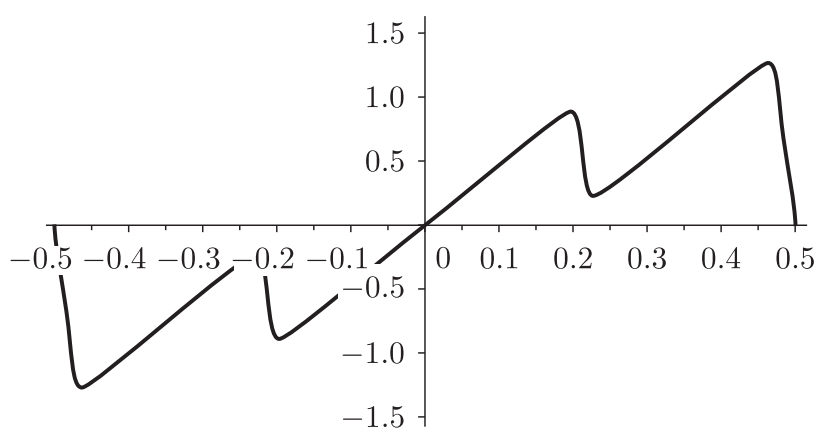

Рис. 3. Типичное решение уравнения Бюргерса

Далее, дадим более подробное описание $N$-волнового поведения. Для типичного начального условия $u_{0}$ (т. е. такого, что $\max \left|u_{0}\right| \sim 1$ и $\max \left|\left(u_{0}\right)_{x}\right| \sim 1$ ) и при $t>1 /\left(\min \left(u_{0}\right)_{x}\right), t \sim 1$, численные расчеты свидетельствуют [2], что типичное решение $u(t, \cdot)$ уравнения с вязкостью обладает следующими свойствами (см. рис. 3):

- Амплитуда решения: $~ 1$.

- Число обрывов на период: $\sim 1$.

- "Размер вертикального спадания" на уровне обрыва: -1.

- "Ширина" обрыва: $\sim \nu$. 
Теперь под $S_{p}(\ell)$ мы будем понимать структурную функцию, определяемую формулой

$$
S_{p}(\ell)=\int_{S^{1}}|u(x+\ell)-u(x)|^{p} d x, \quad p \geqslant 1 .
$$

При $\ell \in \mathbf{J}_{\text {inert }}$ величина $\ell$ обычно меньше, чем интервал между двумя обрывами, но больше, чем ширина обрыва. Э. Орелл, У. Фриш, Дж. Луцко и М. Вергассола [2] отмечают, что для интервала $[x, x+\ell]$ имеются следующие три возможности.

- $[x, x+\ell]$ покрывает значительную часть обрыва. Вероятность этого равна $C \ell$. При этом

$$
u(x+\ell)-u(x)=\underbrace{-C}_{\text {обрыв }}+\underbrace{C \ell}_{\text {пандусы }}=-C, \quad|u(x+\ell)-u(x)|^{p} \stackrel{p}{\sim} 1 .
$$

- $[x, x+\ell]$ покрывает малую часть обрыва. Вклад от такого члена пренебрежимо мал.

- $[x, x+\ell]$ не пересекается с обрывом. Вероятность этого равна $1-C \ell=C$. При этом

$$
u(x+\ell)-u(x)=\underbrace{C \ell}_{\text {пандусы }}, \quad|u(x+\ell)-u(x)|^{p} \stackrel{p}{\sim} \ell^{p} .
$$

Таким образом,

$$
S_{p}(\ell) \stackrel{p}{\sim} \ell+\ell^{p} \stackrel{p}{\sim} \begin{cases}\ell^{p}, & 0 \leqslant p \leqslant 1 \\ \ell, & p \geqslant 1 .\end{cases}
$$

Иными словами, если $p \geqslant 0$, то из приведенного выше описания следует, что при $\ell \in \mathbf{J}_{\text {inert }}$ структурные функции ведут себя следующим образом:

$$
S_{p}(\ell) \stackrel{p}{\sim} \begin{cases}\ell^{p}, & 0 \leqslant p \leqslant 1 \\ \ell, & p \geqslant 1\end{cases}
$$

Как следствие, если $\ell$ находится в инерционном интервале, то эксцесс $F(\ell)$ ведет себя как $\ell^{-1}$.

Отметим, что асимптотически коэффициенты Фурье $N$-волны удовлетворяют неравенству $|\widehat{u}(k)| \sim k^{-1}$. Отсюда естественно предположить, что при малых $\nu$ и при $k$ из определенного интервала (длина интервала зависит от $k$ ) величины энергетического типа $|\widehat{u}(k)|^{2} / 2$ ведут себя в среднем как $k^{-2}$. А именно, при $k^{-1} \in \mathbf{J}_{\text {inert }}$ физические предсказания дают $E(k) \sim k^{-2}$ с тем же определением для $E(k)$, что и выше, с точностью до отсутствия скобок $\langle\cdot\rangle$ [15], [25], [32], [37]. Ограничение $k^{-1} \in \mathbf{J}_{\text {inert }}$ возникает из-за простых размерностных соображений, $\nu$ - натуральный малый масштаб (ширина обрыва) решений решений (см. рис. 4).

С 1980-х годов началось интенсивное изучение уравнение Бюргерса с различными вариантами случайных возмущений. Наиболее изучен случай с шумом, аддитивным по времени и более или менее гладким в пространстве. Здесь мы рассматриваем только случай, когда шум является $C^{\infty}$-гладким в пространстве (по поводу общего случая мы отсылаем читателя к обзорам [27], [6]). В данной 


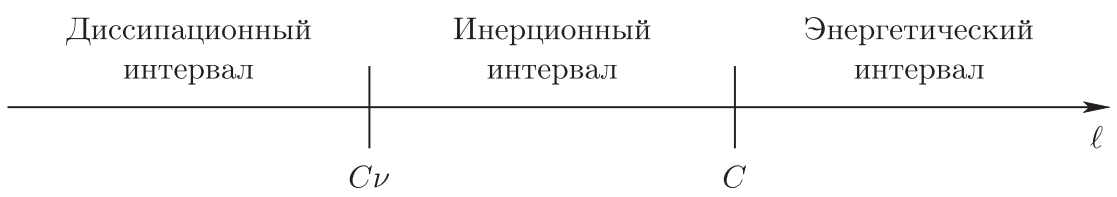

Рис. 4. Пространственные масштабы для уравнения Бюргерса

ситуации численное моделирование и физические предсказания дают в точности те же самые результаты, что и в детерминированном случае, с той лишь разницей, что мы рассматриваем математические ожидания величин [29]. Эвристически это объясняется тем, что возмущение действует на больших масштабах, в энергетическом интервале, и, таким образом, оказывает влияние на мелкомасштабном уровне лишь неявно, как источник энергии.

\section{3. Основные результаты}

В разделе 4 мы рассмотрим детерминированное уравнение Бюргерса. Для начала в п. 4.1 мы установим точные оценки сверху и снизу для некоторых соболевских норм решения $u$. Лемма 4.1 содержит ключевую оценку

$$
u_{x}(t, x) \leqslant \min \left(D, \sigma^{-1} t^{-1}\right)
$$

(по поводу определения $D$ см. (15)). Основные результаты о соболевских нормах решений приведены в теореме 4.8. Именно, если $m=0,1, p \in[1, \infty]$ или $m \geqslant 2, p \in(1, \infty]$, то

$$
\left(\left\{|u(t)|_{m, p}^{\alpha}\right\}\right)^{1 / \alpha} \stackrel{m, p, \alpha}{\sim} \nu^{-\gamma}, \quad \alpha>0
$$

где $\{\cdot\}$ обозначает усреднение по времени на интервале $\left[T_{1}, T_{2}\right]$ (см. (38)). Напомним, что $\gamma(m, p)=\max (0, m-1 / p)$.

В п. 4.2 мы получим точные оценки для аналогов величин, характеризующих гидродинамическую турбулентность. Ниже мы предполагаем, что $\nu \in\left(0, \nu_{0}\right]$, где $\nu_{0} \in(0,1]$ зависит только от $f$ и $D$. Для начала определим непустые попарно непересекающиеся интервалы

$$
J_{1}=\left(0, C_{1} \nu\right], \quad J_{2}=\left(C_{1} \nu, C_{2}\right], \quad J_{3}=\left(C_{2}, 1\right]
$$

(по поводу определений $\nu_{0}, C_{1}$ и $C_{2}$ см. (51); данные величины зависят только от $f$ и $D)$. Используя $(25)$ и $(26)$, мы доказываем в теореме 4.17 , что при $\ell \in J_{1}$

$$
S_{p}(\ell) \stackrel{p}{\sim} \begin{cases}\ell^{p}, & 0 \leqslant p \leqslant 1 \\ \ell^{p} \nu^{-(p-1)}, & p \geqslant 1,\end{cases}
$$

а при $\ell \in J_{2}$

$$
S_{p}(\ell) \stackrel{p}{\sim} \begin{cases}\ell^{p}, & 0 \leqslant p \leqslant 1 \\ \ell, & p \geqslant 1\end{cases}
$$


Как следствие, при $\ell \in J_{2}$ для эксцесса $F(\ell)$ имеет место оценка

$$
F(\ell)=\frac{S_{4}(\ell)}{S_{2}^{2}(\ell)} \sim \ell^{-1} .
$$

Окончательно, мы получаем оценки для спектральной асимптотики бургулентности. С одной стороны, как следствие из теоремы 4.8, при $m \geqslant 1$ мы получаем

$$
\left\{|\widehat{u}(k)|^{2}\right\} \stackrel{m}{\lesssim} k^{-2 m}\|u\|_{m}^{2} \stackrel{m}{\lesssim}(k \nu)^{-2 m} \nu .
$$

В частности, $\left\{|\widehat{u}(k)|^{2}\right\}$ убывает быстрее любой степени при $|k| \succeq \nu^{-1}$. С другой стороны, по теореме 4.21 , если $k$ таково, что $k^{-1} \in J_{2}$, то для энергетического спектра выполнено соотношение

$$
E(k)=\frac{\sum_{|n| \in\left[M^{-1} k, M k\right]}\left\langle|\widehat{u}(n)|^{2}\right\rangle}{\sum_{|n| \in\left[M^{-1} k, M k\right]} 1} \sim k^{-2},
$$

где $M \geqslant 1$ зависит только от $f$ и $D$.

Следует отметить, что данные результаты дают строгое обоснование физических предсказаний, изложенных в п. 2.2. Более того, при этом отпадает необходимость усреднения начального условия (что, к примеру, осуществлено в [2]). Это объясняется специфической структурой детерминированного обобщенного уравнения Бюргерса: начальное условие $u_{0}$ является таким же общим, как и отношение порядков $\left(u_{0}\right)_{x}$ и $u_{0}$, которое можно оценить сверху с использованием величины $D$.

Результаты раздела 5 могут быть сформулированы в точно таком же виде, за исключением следующих двух моментов.

- Оценки выполнены равномерно по $t$ (при достаточно больших $t$ ) и $u_{0}$. С другой стороны, все оцениваемые величины должны быть заменены их математическими ожиданиями. В частности, мы модифицируем значение скобок $\{\cdot\}$.

- Зависимость от $D$ заменяется на зависимость от статистических свойств возмущения.

В разделе 6 мы приводим результаты о существовании и единственности стационарной меры для обобщенного уравнения Бюргерса со случайным возмущением. Из данных результатов вытекает, что все оценки, упомянутые в разделе 5 , также имеют место при замене математического ожидания и усреднения по времени на усреднение по стационарной мере $\mu$.

\section{4. Оценки в невозмущенном случае}

Результаты п. 4.1 были получены А. Э. Бирюком [7] в слегка отличающемся виде для соболевских норм в пространствах $H^{m}, m \geqslant 1$. В нашем изложении мы следуем работе [9], где, обобщая результаты Бирюка, автором получены оценки соболевских норм пространства $W^{m, p}$ при $m \in\{0,1\}, p \in[1, \infty]$ или при $m \geqslant 2, p \in(1, \infty]$ с использованием неравенства Гёльдера и неравенства Гальярдо-Ниренберга. В [7] Бирюк также установил спектральные оценки 
сверху и снизу, что позволило ему найти правильную асимптотику для масштаба длины диссипации при $\nu \rightarrow 0$. Данные результаты уточнены в [9], где были получены точные оценки для структурных функций. В п. 4.2 мы приводим только результаты из [9]; для сравнения их с результатами из [7] читатель должен обратиться к [9].

4.1. Оценки соболевских норм. Для начала напомним доказательство ключевой оценки сверху для $u_{x}$, являющейся переформулировкой принщипа максимума Кружкова [40].

Лемма 4.1. Имеет место следующая оценка:

$$
u_{x}(t, x) \leqslant \min \left(D, \sigma^{-1} t^{-1}\right) .
$$

ДокАзАтЕльство. Дифференцируя уравнение (1) один раз в пространстве, имеем

$$
\left(u_{x}\right)_{t}+f^{\prime \prime}(u) u_{x}^{2}+f^{\prime}(u)\left(u_{x}\right)_{x}=\nu\left(u_{x}\right)_{x x} .
$$

Теперь рассмотрим точку $\left(t_{1}, x_{1}\right)$, в которой $u_{x}$ достигает максимума на цилиндре $S=[0, t] \times S^{1}$. Предположим, что $t_{1}>0$ и что этот максимум неотрицателен. По формуле Тейлора вытекает, что в этой точке $\left(u_{x}\right)_{t} \geqslant 0,\left(u_{x}\right)_{x}=0$ и $\left(u_{x}\right)_{x x} \leqslant 0$. Следовательно, так как $f^{\prime \prime}(u) \geqslant \sigma$ по $(2)$, то из (27) вытекает, что $\sigma u_{x}^{2} \leqslant 0$, что невозможно. Таким образом, $u_{x}$ может иметь неотрицательный максимум на $S$ только при $t_{1}=0$. Иными словами, так как $\left(u_{0}\right)_{x}$ имеет нулевое среднее, то

$$
u_{x}(t, x) \leqslant \max _{x \in S^{1}}\left(u_{0}\right)_{x}(x) \leqslant D .
$$

Неравенство

$$
u_{x}(t, x) \leqslant \sigma^{-1} t^{-1}
$$

установлено в [40] при помощи аналогичного принципа максимума, примененного к функции $v=t u_{x}$. Действительно, такая функция может иметь неотрицательный максимум на $S$ в точке $\left(t_{1}, x_{1}\right)$ только при $t_{1}>0$. Умножая $(27)$ на $t^{2}$, мы получаем

$$
t \underbrace{v_{t}}_{\geqslant 0}+t f^{\prime}(u) \underbrace{v_{x}}_{0}+\left(-v+f^{\prime \prime}(u) v^{2}\right)=\nu t \underbrace{v_{x x}}_{\leqslant 0} .
$$

Таким образом, $v \leqslant \sigma^{-1}$ на $S$. Иными словами, $u_{x} \leqslant \sigma^{-1} t^{-1}$ при всех $t>0$. Лемма доказана.

Поскольку средние по пространству $u(t)$ и $u_{x}(t)$ равны нулю при всех $t$, то мы получаем следующие оценки сверху:

$$
\begin{gathered}
|u(t)|_{p} \leqslant|u(t)|_{\infty} \leqslant \int_{S^{1}} u_{x}^{+}(t) d x \leqslant \min \left(D, \sigma^{-1} t^{-1}\right), \quad 1 \leqslant p \leqslant+\infty \\
|u(t)|_{1,1}=2 \int_{S^{1}} u_{x}^{+}(t) d x \leqslant 2 \min \left(D, \sigma^{-1} t^{-1}\right) .
\end{gathered}
$$

Напомним стандартную оценку [12] для нелинейности

$$
\left\langle v^{(m+1)},(f(v))^{(m)}\right\rangle .
$$


Лемма 4.2. Пусть $v \in C^{\infty},|v|_{\infty} \leqslant N$. Тогда

$$
N_{m}(v)=\left|\left\langle v^{(m+1)},(f(v))^{(m)}\right\rangle\right| \stackrel{m, N}{\lesssim}\|v\|_{m}\|v\|_{m+1}, \quad m \geqslant 1 .
$$

ДокАЗАТЕЛЬство. Зафиксируем $m \geqslant 1$. В доказательстве константы, обозначаемые через $\widetilde{C}$, будут зависеть только от $m, N$. Имеем

$$
\begin{aligned}
& N_{m}(v) \leqslant \widetilde{C} \sum_{k=1}^{m} \sum_{\substack{1 \leqslant a_{1} \leqslant \cdots \leqslant a_{k} \leqslant m \\
a_{1}+\cdots+a_{k}=m}} \int_{S^{1}}\left|v^{(m+1)} v^{\left(a_{1}\right)} \cdots v^{\left(a_{k}\right)} f^{(k)}(v)\right| \\
& \leqslant \widetilde{C} \max _{x \in[-N, N]} \max \left(f^{\prime}(x), \ldots, f^{(m)}(x)\right) \\
& \times \sum_{k=1}^{m} \sum_{\substack{1 \leqslant a_{1} \leqslant \cdots \leqslant a_{k} \leqslant m \\
a_{1}+\cdots+a_{k}=m}} \int_{S^{1}}\left|v^{\left(a_{1}\right)} \cdots v^{\left(a_{k}\right)} v^{(m+1)}\right| .
\end{aligned}
$$

Воспользовавшись (13), неравенством Гёльдера и неравенством Гальярдо-Ниренберга, мы получаем

$$
\begin{aligned}
N_{m}(v) \leqslant & \widetilde{C}(1+N)^{\max (h(1), \ldots, h(m))} \\
& \times \sum_{k=1}^{m} \sum_{\substack{1 \leqslant a_{1} \leqslant \cdots \leqslant a_{k} \leqslant m \\
a_{1}+\cdots+a_{k}=m}} \int_{S^{1}}\left|v^{\left(a_{1}\right)} \cdots v^{\left(a_{k}\right)} v^{(m+1)}\right| \\
\leqslant & \widetilde{C} \sum_{k=1}^{m} \sum_{\substack{1 \leqslant a_{1} \leqslant \cdots \leqslant a_{k} \leqslant m \\
a_{1}+\cdots+a_{k}=m}}\left(\left|v^{\left(a_{1}\right)}\right|_{2 m / a_{1}} \cdots\left|v^{\left(a_{k}\right)}\right|_{2 m / a_{k}}\|v\|_{m+1}\right) \\
\leqslant & \widetilde{C}\|v\|_{m+1} \sum_{\substack { m=1 \\
\begin{subarray}{c}{1 \leqslant a_{1} \leqslant \cdots \leqslant a_{k} \leqslant m \\
a_{1}+\cdots+a_{k}=m{ m = 1 \\
\begin{subarray} { c } { 1 \leqslant a _ { 1 } \leqslant \cdots \leqslant a _ { k } \leqslant m \\
a _ { 1 } + \cdots + a _ { k } = m } }\end{subarray}}\left(\left(\|v\|_{m}^{a_{1} / m}|v|_{\infty}^{\left(m-a_{1}\right) / m}\right) \times \cdots\right. \\
\leqslant & \left.\times\left(\|v\|_{m}^{a_{k} / m}|v|_{\infty}^{\left(m-a_{k}\right) / m}\right)\right) \\
& \widetilde{C}(1+N)^{m-1}\|v\|_{m}\|v\|_{m+1}=\widetilde{C}\|v\|_{m}\|v\|_{m+1} .
\end{aligned}
$$

Лемма доказана.

В следующем результате показывается существование сильного нелинейного затухания, которое не дает последовательным производным $и$ стать слишком большими.

Лемма 4.3. Имеет место следующее неравенство:

$$
\|u(t)\|_{1}^{2} \lesssim \nu^{-1}
$$

Если $m \geqslant 2, m o$

$$
\|u(t)\|_{m}^{2} \stackrel{m}{\lesssim} \max \left(\nu^{-(2 m-1)}, t^{-(2 m-1)}\right) .
$$


ДокАЗАТЕЛьСтво. Зафиксируем $m \geqslant 1$ и обозначим

$$
x(t)=\|u(t)\|_{m}^{2} .
$$

Мы покажем, что верна следующая импликация:

$$
x(t) \geqslant C^{\prime} \nu^{-(2 m-1)} \Longrightarrow \frac{d}{d t} x(t) \leqslant-(2 m-1) x(t)^{2 m /(2 m-1)},
$$

где $C^{\prime}$ - фиксированное положительное число, которое мы выберем позже. Ниже все константы $C$ не зависят от $C^{\prime}$.

Итак, предположим, что $x(t) \geqslant C^{\prime} \nu^{-(2 m-1)}$. Интегрируя по частям в пространстве и используя (28) (с $p=\infty$ ) и лемму 4.2, мы получаем следующее соотношение для диссипации энергии:

$$
\begin{aligned}
\frac{d}{d t} x(t) & =-2 \nu\|u(t)\|_{m+1}^{2}+2\left\langle u^{(m+1)}(t),(f(u(t)))^{(m)}\right\rangle \\
& \leqslant-2 \nu\|u(t)\|_{m+1}^{2}+C\|u(t)\|_{m}\|u(t)\|_{m+1} .
\end{aligned}
$$

Применяя неравенство Гальярдо-Ниренберга к $u_{x}$ и затем используя (29), мы имеем

$$
\begin{aligned}
\|u(t)\|_{m} & \leqslant C\|u(t)\|_{m+1}^{(2 m-1) /(2 m+1)}|u(t)|_{1,1}^{2 /(2 m+1)} \\
& \leqslant C\|u(t)\|_{m+1}^{(2 m-1) /(2 m+1)}
\end{aligned}
$$

Таким образом,

$$
\frac{d}{d t} x(t) \leqslant\left(-2 \nu\|u(t)\|_{m+1}^{2 /(2 m+1)}+C\right)\|u(t)\|_{m+1}^{4 m /(2 m+1)} .
$$

Из (32) следует, что

$$
\|u(t)\|_{m+1}^{2 /(2 m+1)} \geqslant C x(t)^{1 /(2 m-1)}
$$

а поскольку по предположению $x(t) \geqslant C^{\prime} \nu^{-(2 m-1)}$, то

$$
\|u(t)\|_{m+1}^{2 /(2 m+1)} \geqslant C\left(C^{\prime}\right)^{1 /(2 m-1)} \nu^{-1} .
$$

Комбинируя неравенства (33)-(35), при достаточно больших $C^{\prime}$ получаем

$$
\frac{d}{d t} x(t) \leqslant\left(-C\left(C^{\prime}\right)^{1 /(2 m-1)}+C\right) x(t)^{2 m /(2 m-1)} .
$$

Это показывает, что мы можем выбрать $C^{\prime}$ таким образом, чтобы импликация (30) имела место.

При $m=1$ из (15) и (30) получаем

$$
x(t) \leqslant \max \left(C^{\prime} \nu^{-1}, D^{2}\right) \leqslant \max \left(C^{\prime}, D^{2}\right) \nu^{-1}, \quad t \geqslant 0 .
$$


Теперь рассмотрим случай $m \geqslant 2$. Покажем, что

$$
x(t) \leqslant \max \left(C^{\prime} \nu^{-(2 m-1)}, t^{-(2 m-1)}\right) .
$$

Действительно, если $x(s) \leqslant C^{\prime} \nu^{-(2 m-1)}$ при некотором $s \in[0, t]$, то из (30) следует, что $x(s)$ останется ниже порогового значения до момента времени $t$.

Теперь предположим, что $x(s)>C^{\prime} \nu^{-(2 m-1)}$ при всех $s \in[0, t]$. Обозначим

$$
\widetilde{x}(s)=(x(s))^{-1 /(2 m-1)}, \quad s \in[0, t] .
$$

Из $(30)$ имеем $d \widetilde{x}(s) / d s \geqslant 1$. Как следствие, $\widetilde{x}(t) \geqslant t$ и $x(t) \leqslant t^{-(2 m-1)}$. Таким образом, в нашей ситуации неравенство (36) по-прежнему имеет место. Это завершает доказательство леммы.

Применяя неравенство Гальярдо-Ниренберга к $u^{(m)}$, мы получаем следующее неравенство при $m \geqslant 1$ :

$$
|u(t)|_{m, \infty} \lesssim\|u(t)\|_{m}^{1 / 2}\|u(t)\|_{m+1}^{1 / 2} \stackrel{m}{\lesssim} \max \left(\nu^{-m}, t^{-m}\right) .
$$

Аналогично, обращаясь к неравенству Гальярдо-Ниренберга и интерполируя между $|u|_{1,1}$ и $\|u\|_{M}$ при больших значениях $M$, мы приходим к следующему результату (напомним, что $\gamma=\max (0, m-1 / p)$ ).

Tеорема 4.4. Пусть $m \in\{0,1\}, p \in[1, \infty]$ или $m \geqslant 2, p \in(1, \infty]$. Тогда

$$
\left(|u(t)|_{m, p}^{\alpha}\right)^{1 / \alpha} \stackrel{m, p, \alpha}{\lesssim} \max \left(t^{-\gamma}, \nu^{-\gamma}\right), \quad \alpha>0 .
$$

Введем следующие величины:

$$
T_{1}=\frac{1}{4} D^{-2} \widetilde{C}^{-1}, \quad T_{2}=\max \left(\frac{3}{2} T_{1}, 2 D \sigma^{-1}\right),
$$

где $\widetilde{C}$ - константа такая, что $\|u(t)\|_{1}^{2} \leqslant \widetilde{C} \nu^{-1}$ для всех $t$ (ср. с леммой 4.3).

Здесь и ниже, если $A(\cdot)$ - зависящий от времени функционал со значениями в пространстве Соболева, то через $\{A(t)\}$ мы обозначаем усреднение по времени:

$$
\{A(t)\}=\frac{1}{T_{2}-T_{1}} \int_{T_{1}}^{T_{2}} A(t) .
$$

Лемма 4.5. Имеет место следующее неравенство:

$$
\left\{\|u(t)\|_{1}^{2}\right\} \gtrsim \nu^{-1} .
$$

ДокАЗАТЕЛЬство. Интегрируя по частям в пространстве, мы получаем следующее тождество для диссипации:

$$
\frac{d}{d t}|u(t)|^{2}=\int_{S^{1}}\left(-2 u f^{\prime}(u) u_{x}+2 \nu u u_{x x}\right)=-2 \nu\|u(t)\|_{1}^{2} .
$$

Далее, интегрируя по времени и воспользовавшись (15) и леммой 4.3, мы получаем

$$
\left|u\left(T_{1}\right)\right|^{2}=\left|u_{0}\right|^{2}-2 \nu \int_{0}^{T_{1}}\|u(t)\|_{1}^{2} \geqslant D^{-2}-2 T_{1} \widetilde{C} \geqslant \frac{1}{2} D^{-2} .
$$


Отсюда, интегрируя (39) по времени и используя (28) (с $p=2)$, имеем

$$
\begin{aligned}
\left\{\|u(t)\|_{1}^{2}\right\} & =\frac{1}{2 \nu\left(T_{2}-T_{1}\right)}\left(\left|u\left(T_{1}\right)\right|^{2}-\left|u\left(T_{2}\right)\right|^{2}\right) \\
& \geqslant \frac{1}{2 \nu\left(T_{2}-T_{1}\right)}\left(\frac{1}{2} D^{-2}-\sigma^{-2} T_{2}^{-2}\right) \\
& \geqslant \frac{D^{-2}}{8\left(T_{2}-T_{1}\right)} \nu^{-1},
\end{aligned}
$$

что доказывает лемму.

Усредненная по времени оценка снизу дает аналогичные оценки для соболевских норм.

Лемма 4.6. Пусть $m \geqslant 1$. Тогда

$$
\left\{\|u(t)\|_{m}^{2}\right\} \stackrel{m}{\gtrsim} \nu^{-(2 m-1)}
$$

ДокАЗАТЕЛЬство. Случай $m=1$ покрывается предыдущей леммой, так что мы можем предположить, что $m \geqslant 2$. Воспользовавшись $(29)$ и неравенством Гальярдо-Ниренберга, имеем

$$
\left\{\|u(t)\|_{m}^{2}\right\} \stackrel{m}{\gtrsim}\left\{\|u(t)\|_{m}^{2}|u(t)|_{1,1}^{4 m-4}\right\} \stackrel{m}{\gtrsim}\left\{\|u(t)\|_{1}^{4 m-2}\right\} .
$$

Окончательно, по неравенству Гёльдера и лемме 4.5

$$
\left\{\|u(t)\|_{m}^{2}\right\} \stackrel{m}{\gtrsim}\left\{\|u(t)\|_{1}^{4 m-2}\right\} \stackrel{m}{\gtrsim}\left\{\|u(t)\|_{1}^{2}\right\}^{2 m-1} \stackrel{m}{\gtrsim} \nu^{-(2 m-1)} .
$$

Лемма 4.6 доказана.

Доказательство следующей леммы аналогично.

Лемма 4.7. Пусть $m \geqslant 0, p \in[1, \infty]$. Тогда

$$
\left\{|u(t)|_{m, p}^{2}\right\} \stackrel{m, p}{\gtrsim} \nu^{-2 \gamma} .
$$

Следующая теорема подытоживает все результаты данного пункта, которые нам потребуются ниже, за исключением леммы 4.1.

Tеорема 4.8. Пусть $m \in\{0,1\}, p \in[1, \infty]$ или $m \geqslant 2, p \in(1, \infty]$. Тогда

$$
\left(\left\{|u(t)|_{m, p}^{\alpha}\right\}\right)^{1 / \alpha} \stackrel{m, p, \alpha}{\sim} \nu^{-\gamma}, \quad \alpha>0
$$

где $\{\cdot\}$ обозначает усреднение по времени на $\left[T_{1}, T_{2}\right]$. Оценка сверху в (40) имеет место без усреднения по времени, равномерно по $t$, отделенным от 0 . Именно,

$$
|u(t)|_{m, p} \stackrel{m, p}{\lesssim} \max \left(\nu^{-\gamma}, t^{-\gamma}\right) .
$$

Оценки снизу верны при всех $m \geqslant 0, p \in[1, \infty], \alpha>0$. 
ДокАЗАтЕЛьство. Оценки сверху следуют из теоремы 4.4. Для $\alpha \geqslant 2$ оценки снизу вытекают из леммы 4.7 и неравенства Гёльдера. При $m=0, p \in[1, \infty]$ и при $m \geqslant 1, p \in(1, \infty]$ оценки снизу для $\alpha \in(0,2)$ получаются из оценок снизу для $\alpha=2$, оценок сверху для $\alpha=3$ и неравенства Гёльдера. Действительно,

$$
\begin{aligned}
\left\{|u(t)|_{m, p}^{\alpha}\right\} & \geqslant\left(\left\{|u(t)|_{m, p}^{2}\right\}\right)^{3-\alpha}\left(\left\{|u(t)|_{m, p}^{3}\right\}\right)^{-(2-\alpha)} \\
& \gtrsim \nu^{-(6-2 \alpha) \gamma} \nu^{(6-3 \alpha) \gamma}=\nu^{-\alpha \gamma} .
\end{aligned}
$$

Для $|u|_{m, 1}, m>1$, оценки снизу вытекают из соответствующих оценок для $|u|_{m-1, \infty}$. Теорема доказана.

При $p=2, \alpha=2$ теорема 4.8 утверждает, что при целых $m \geqslant 1$

$$
\left\{\|u\|_{m}^{2}\right\} \stackrel{m}{\sim} \nu^{-(2 m-1)}
$$

При помощи стандартных интерполяционных рассуждений можно показать, что оценка сверху в (41) выполнена также для нецелых индексов $s>1$. В действительности, это верно и для оценки снизу, поскольку для любого целого $n>s$

$$
\left\{\|u\|_{s}^{2}\right\} \geqslant\left\{\|u\|_{n}^{2}\right\}^{n-s+1}\left\{\|u\|_{n+1}^{2}\right\}^{-(n-s)} \stackrel{s}{\gtrsim} \nu^{-(2 s-1)} .
$$

4.2. Оценки для мелкомасштабных величин. В данном пункте мы изучаем аналоги величин, важных для исследования гидродинамической турбулентности. Данные величины будут рассматриваться как в физическом пространстве (структурные функции), так и в пространстве Фурье (энергетический спектр). Предположив, что $\nu \leqslant \nu_{0}$, определим следующие интервалы:

$$
J_{1}=\left(0, C_{1} \nu\right], \quad J_{2}=\left(C_{1} \nu, C_{2}\right], \quad J_{3}=\left(C_{2}, 1\right]
$$

Положительные константы $\nu_{0}, C_{1}, C_{2}$ будут выбраны в $(50),(51)$ таким образом, чтобы выполнялись неравенства $C_{1} \nu_{0}<C_{2}<1$. Это гарантирует, что интервалы $J_{i}$ непусты и не пересекаются.

Из теоремы 4.8 мы имеем, что $\left\{|u|^{2}\right\} \sim 1$. С другой стороны, интегрируя по частям, мы получаем из (29), что

$$
\begin{aligned}
\left\{|\widehat{u}(n)|^{2}\right\} & =(2 \pi n)^{-2}\left\{\left|\int_{S^{1}} e^{2 \pi i n x} u_{x}(x) d x\right|^{2}\right\} \\
& \leqslant(2 \pi n)^{-2}\left\{|u|_{1,1}^{2}\right\} \leqslant C n^{-2}
\end{aligned}
$$

при этом $C_{1}$ и $C_{2}$ могут быть сделаны произвольно малыми (ср. (52)). Как следствие, часть суммы $\left\{\sum|\widehat{u}(n)|^{2}\right\}$, содержащаяся в фурье-модах, соответствующих $J_{3}$, может быть сделана сколь угодно большой. $\mathrm{K}$ примеру, можно предположить, что

$$
\left\{\sum_{|n|<C_{2}^{-1}}|\widehat{u}(n)|^{2}\right\} \geqslant \frac{99}{100}\left\{\sum_{n \in \mathbb{Z}}|\widehat{u}(n)|^{2}\right\} .
$$


При $p \geqslant 0$ определим структурную функцию порядка $p$ следующим образом:

$$
S_{p}(\ell)=\left\{\int_{S^{1}}|u(t, x+\ell)-u(t, x)|^{p} d x\right\} .
$$

Эксцесс $F(\ell)$, измеряющий пространственную перемежаемость, определяется формулой

$$
F(\ell)=\frac{S_{4}(\ell)}{S_{2}^{2}(\ell)}
$$

Окончательно, при $k \geqslant 1$ определим энергетический спектр (усредненный по слоям):

$$
E(k)=\left\{\frac{\sum_{|n| \in\left[M^{-1} k, M k\right]}|\widehat{u}(n)|^{2}}{\sum_{|n| \in\left[M^{-1} k, M k\right]} 1}\right\},
$$

где $M \geqslant 1$ - константа, которую мы выберем позднее (см. доказательство теоремы 4.21).

Мы начнем с оценки функции $S_{p}(\ell)$ сверху.

Лемма 4.9. Пусть $\ell \in[0,1]$. Тогда

$$
S_{p}(\ell) \stackrel{p}{\lesssim} \begin{cases}\ell^{p}, & 0 \leqslant p \leqslant 1 \\ \ell^{p} \nu^{-(p-1)}, & p \geqslant 1\end{cases}
$$

ДокАЗАТЕЛЬСтво. Сначала рассмотрим случай $p \geqslant 1$. Имеем

$$
\begin{aligned}
S_{p}(\ell) & =\left\{\int_{S^{1}}|u(x+\ell)-u(x)|^{p} d x\right\} \\
& \leqslant\left\{\left(\int_{S^{1}}|u(x+\ell)-u(x)| d x\right)\left(\max _{x}|u(x+\ell)-u(x)|^{p-1}\right)\right\} .
\end{aligned}
$$

Из тождества $\int_{S^{1}}(u(\cdot+\ell)-u(\cdot))=0$ и неравенства Гёльдера получаем

$$
\begin{aligned}
S_{p}(\ell) \leqslant & \left\{\left(2 \int_{S^{1}}(u(x+\ell)-u(x))^{+} d x\right)^{p}\right\}^{1 / p} \\
& \times\left\{\max _{x}|u(x+\ell)-u(x)|^{p}\right\}^{(p-1) / p} \\
\leqslant & C \ell\left\{\max _{x}|u(x+\ell)-u(x)|^{p}\right\}^{(p-1) / p}
\end{aligned}
$$

где второе неравенство следует из леммы 4.1. Окончательно, по теореме 4.8

$$
S_{p}(\ell) \leqslant C \ell\left\{\left(\ell|u|_{1, \infty}\right)^{p}\right\}^{(p-1) / p} \leqslant C \ell^{p} \nu^{-(p-1)} .
$$


Случай $p<1$ непосредственно вытекает из случая $p=1$, поскольку тогда $S_{p}(\ell) \leqslant\left(S_{1}(\ell)\right)^{p}$, что следует из неравенства Гёльдера. Лемма доказана.

Если $\ell \in J_{2} \cup J_{3}$, то мы можем получить более точную оценку при $p \geqslant 1$.

Лемма 4.10. Пусть $\ell \in J_{2} \cup J_{3}$. Тогда

$$
S_{p}(\ell) \stackrel{p}{\lesssim} \begin{cases}\ell^{p}, & 0 \leqslant p \leqslant 1 \\ \ell, & p \geqslant 1 .\end{cases}
$$

ДокАЗАТЕЛЬСтво. В данной ситуации рассуждения практически идентичны использованным в доказательстве предыдущей леммы. Единственным отличием является то, что мы используем другую оценку для правой части неравенства (45). Именно, по теореме 4.8

$$
\begin{aligned}
S_{p}(\ell) & \leqslant C \ell\left\{\max _{x}|u(x+\ell)-u(x)|^{p}\right\}^{(p-1) / p} \\
& \leqslant C \ell\left\{\left(2|u|_{\infty}\right)^{p}\right\}^{(p-1) / p} \leqslant C \ell .
\end{aligned}
$$

Лемма доказана.

ЗАмЕчАниЕ 4.11. В действительности, утверждения лемм 4.9 и 4.10 имеют место даже в случае, если мы откажемся от усреднения по времени, поскольку при их выводе мы пользовались лишь оценками сверху, которые выполняются равномерно по $t \geqslant T_{1}$.

Для доказательства оценок снизу для $S_{p}(\ell)$ нам потребуется еще одна лемма. Грубо говоря, эта лемма утверждает, что найдется достаточно большое множество $L_{K} \subset\left[T_{1}, T_{2}\right]$ такое, что при $t \in L_{K}$ ряд соболевских норм имеют такой же порядок, как и их усреднения по времени. Таким образом, при $t \in L_{K}$ мы можем доказать существование обрыва высоты $\geqslant C$ и длины $\geqslant C \nu$. При этом мы используем некоторые рассуждения из [2] (см. также п. 2.2).

Отметим, что в следующем определении предположения (46), (47) содержат оценки снизу и сверху, а предположение (48) содержит только оценку сверху. Неравенство $|u(t)|_{\infty} \leqslant \max u_{x}(t)$ в (46) выполнено всегда, поскольку $u(t)$ имеет нулевое среднее значение, а длина $S^{1}$ равна 1.

ОПРЕДЕЛЕНИЕ 4.12. Пусть $K>1$. Через $L_{K}$ мы обозначим множество всех $t \in\left[T_{1}, T_{2}\right]$, для которых выполнены следующие предположения:

$$
\begin{gathered}
K^{-1} \leqslant|u(t)|_{\infty} \leqslant \max u_{x}(t) \leqslant K, \\
K^{-1} \nu^{-1} \leqslant|u(t)|_{1, \infty} \leqslant K \nu^{-1} \\
|u(t)|_{2, \infty} \leqslant K \nu^{-2}
\end{gathered}
$$

Лемма 4.13. Существуют константы $C, K_{1}>0$ такие, что $\lambda\left(L_{K}\right) \geqslant C$ при $K \geqslant K_{1}\left(\right.$ здесъ $\lambda\left(L_{K}\right)-$ мера Лебега множества $\left.L_{K}\right)$.

ДоказАтельство. Для начала отметим, что если $K \leqslant K^{\prime}$, то $L_{K} \subset L_{K^{\prime}}$. По лемме 4.1 и теореме 4.8 при достаточно больших $K$ оценки сверху в (46)-(48) 
выполнены при всех $t \geqslant T_{1}$. Следовательно, если обозначить через $B_{K}$ множество всех $t$ таких, что

оценки снизу в (46), (47) выполнены для данного $K$,

то нам достаточно доказать утверждение леммы с $L_{K}$, замененным на $B_{K}$. Далее, через $D_{K}$ обозначим множество всех $t$ таких, что

оценка снизу в (47) выполнена для данного $K$.

По неравенству Гальярдо-Ниренберга

$$
|u|_{\infty} \geqslant C|u|_{2, \infty}^{-1}|u|_{1, \infty}^{2} .
$$

Таким образом, если $\omega \in D_{K}$, то выполнено утверждение леммы с $B_{K^{\prime}}$ при достаточно больших $K^{\prime}$. Теперь остается показать, что существует константа $C>0$ такая, что $\lambda\left(D_{K}\right) \geqslant C$ при достаточно больших $K$. Несложно проверить, что

$$
\left\{|u|_{1, \infty} \mathbf{1}\left(|u|_{1, \infty}<K^{-1} \nu^{-1}\right)\right\}<K^{-1} \nu^{-1} .
$$

Здесь $\mathbf{1}(A)$ - характеристическая функция события $A$.

Далее, воспользовавшись оценкой для $\left\{|u|_{1, \infty}^{2}\right\}$ из теоремы 4.8, мы имеем

$$
\left\{|u|_{1, \infty} \mathbf{1}\left(|u|_{1, \infty}>K \nu^{-1}\right)\right\}<K^{-1} \nu\left\{|u|_{1, \infty}^{2}\right\} \leqslant C K^{-1} \nu^{-1} .
$$

Введем функцию

$$
f=|u|_{1, \infty} \mathbf{1}\left(K_{0}^{-1} \nu^{-1} \leqslant|u|_{1, \infty} \leqslant K_{0} \nu^{-1}\right) .
$$

Из приведенных выше неравенств и оценки снизу для $\left\{|u|_{1, \infty}\right\}$, полученной в теореме 4.8, мы имеем

$$
\{f\} \geqslant\left(C-K_{0}^{-1}-C K_{0}^{-1}\right) \nu^{-1} \geqslant C_{0} \nu^{-1}
$$

при некоторых подходящих константах $C_{0}$ и $K_{0}$. Так как $f \leqslant K_{0} \nu^{-1}$, то

$$
\lambda\left(f \geqslant \frac{1}{2} C_{0} \nu^{-1}\right) \geqslant \frac{1}{2} C_{0} K_{0}^{-1}\left(T_{2}-T_{1}\right) .
$$

Итак, поскольку $|u|_{1, \infty} \geqslant f$, то

$$
\lambda\left(|u|_{1, \infty} \geqslant \frac{1}{2} C_{0} \nu^{-1}\right) \geqslant \frac{1}{2} C_{0} K_{0}^{-1}\left(T_{2}-T_{1}\right)
$$

и, как следствие, существуют $C, K_{1}>0$ такие, что $\lambda\left(D_{K}\right) \geqslant C$ при $K \geqslant K_{1}$. Лемма доказана.

Через $O_{K} \subset\left[T_{1}, T_{2}\right]$ обозначим множество, удовлетворяющее условиям (46), (48) из определения множества $L_{K}$ и условию

$$
K^{-1} \nu^{-1} \leqslant-\min u_{x} \leqslant K \nu^{-1},
$$

заменяющему условие (47). 
СледСТвиЕ 4.14. Пусть $K \geqslant K_{1}, \nu<K_{1}^{-2}$. Тогда $\lambda\left(O_{K}\right) \geqslant C$. Здесъ константы $C, K_{1}$ те же, что и в формулировке леммы 4.13.

ДоказАТЕЛЬСтво. При $K=K_{1}, \nu<K_{1}^{-2}$ из оценок (46), (47) следует, что

$$
\max u_{x}(t) \leqslant K_{1}<K_{1}^{-1} \nu^{-1} \leqslant\left|u_{x}(t)\right|_{\infty}, \quad t \in L_{K} .
$$

Таким образом, в этом случае $O_{K}=L_{K}$, что и требуется в утверждении следствия. Если мы будем увеличивать $K$, сохраняя $\nu$ постоянной, то мера множества $O_{K}$ будет увеличиваться, откуда $\lambda\left(O_{K}\right) \geqslant C$ при $K \geqslant K_{1}$ и $\nu<K_{1}^{-2}$. Следствие доказано.

Теперь зафиксируем

$$
K=K_{1}
$$

и определим

$$
\nu_{0}=\frac{1}{6} K^{-2}, \quad C_{1}=\frac{1}{4} K^{-2}, \quad C_{2}=\frac{1}{20} K^{-4} .
$$

В частности, $0<C_{1} \nu_{0}<C_{2}<1$. Таким образом, интервалы $J_{i}$ непусты и не пересекаются при всех $\nu \in\left(0, \nu_{0}\right]$. Всюду мы предполагаем, что константы зависят от $K$.

В действительности, $C_{1}, C_{2}, \nu_{0}$ можно выбрать любыми, при условии, что

$$
C_{1} \leqslant \frac{1}{4} K^{-2}, \quad 5 K^{2} \leqslant \frac{C_{1}}{C_{2}}<\frac{1}{\nu_{0}} .
$$

Лемма 4.15. Пусть $\ell \in J_{1}$. Тогда

$$
S_{p}(\ell) \stackrel{p}{\gtrsim} \begin{cases}\ell^{p}, & 0 \leqslant p \leqslant 1 \\ \ell^{p} \nu^{-(p-1)}, & p \geqslant 1 .\end{cases}
$$

ДокАЗАТЕЛЬство. Согласно следствию 4.14 нам достаточно проверить, что неравенства выполнены равномерно по $t \in O_{K}$ при замене $S_{p}(\ell)$ на

$$
\int_{S^{1}}|u(x+\ell)-u(x)|^{p} d x .
$$

До конца доказательства мы будем предполагать, что $t \in O_{K}$.

Пусть $z$ - самая левая точка на $S^{1}$ (рассматриваемом как $[0,1)$ ), в которой выполнено неравенство $u^{\prime}(z) \leqslant-K^{-1} \nu^{-1}$. Так как $|u|_{2, \infty} \leqslant K \nu^{-2}$, то

$$
u^{\prime}(y) \leqslant-\frac{1}{2} K^{-1} \nu^{-1}, \quad y \in\left[z-\frac{1}{2} K^{-2} \nu, z+\frac{1}{2} K^{-2} \nu\right] .
$$

Иными словами, интервал

$$
\left[z-\frac{1}{2} K^{-2} \nu, z+\frac{1}{2} K^{-2} \nu\right]
$$

соответствует (части) обрыва. 
Случай $p \geqslant 1$. Так как $\ell \leqslant C_{1} \nu=K^{-2} \nu / 4$, то по неравенству Гёльдера

$$
\begin{aligned}
\int_{S^{1}}|u(x+\ell)-u(x)|^{p} d x & \geqslant \int_{z-K^{-2} \nu / 4}^{z+K^{-2} \nu / 4}|u(x+\ell)-u(x)|^{p} d x \\
& \geqslant\left(\frac{K^{-2} \nu}{2}\right)^{1-p}\left(\int_{z-K^{-2} \nu / 4}^{z+K^{-2} \nu / 4}|u(x+\ell)-u(x)| d x\right)^{p} \\
& =C(p) \nu^{1-p}\left(\int_{z-K^{-2} \nu / 4}^{z+K^{-2} \nu / 4}\left(\int_{x}^{x+\ell}\left(-u^{\prime}(y)\right) d y\right) d x\right)^{p} \\
& \geqslant C(p) \nu^{1-p}\left(\int_{z-K^{-2} \nu / 4}^{z+K^{-2} \nu / 4} \frac{1}{2} \ell K^{-1} \nu^{-1} d x\right)^{p}=C(p) \nu^{1-p} \ell^{p}
\end{aligned}
$$

Случай $p<1$. По неравенству Гёльдера

$$
\begin{aligned}
& \int_{S^{1}}|u(x+\ell)-u(x)|^{p} d x \geqslant \int_{S^{1}}\left((u(x+\ell)-u(x))^{+}\right)^{p} d x \\
& \geqslant\left(\int_{S^{1}}\left((u(x+\ell)-u(x))^{+}\right)^{2} d x\right)^{p-1}\left(\int_{S^{1}}(u(x+\ell)-u(x))^{+} d x\right)^{2-p} .
\end{aligned}
$$

Воспользовавшись оценкой сверху из (46), получаем

$$
\int_{S^{1}}|u(x+\ell)-u(x)|^{p} d x \geqslant\left(\int_{S^{1}} \ell^{2} K^{2} d x\right)^{p-1}\left(\int_{S^{1}}(u(x+\ell)-u(x))^{+} d x\right)^{2-p} .
$$

Далее, так как $\int_{S^{1}}(u(\cdot+\ell)-u(\cdot))=0$, то

$$
\begin{aligned}
\int_{S^{1}}|u(x+\ell)-u(x)|^{p} d x \\
\quad \geqslant C(p) \ell^{2(p-1)}\left(\frac{1}{2} \int_{S^{1}}|u(x+\ell)-u(x)| d x\right)^{2-p} \geqslant C(p) \ell^{p}
\end{aligned}
$$

Последнее неравенство следует из случая $p=1$. Лемма доказана.

Доказательство следующей леммы использует один прием из работы [2], который становится количественным, если мы рассматриваем $t \in O_{K}$.

Лемма 4.16. Пусть $m \geqslant 0 u \ell \in J_{2}$. Тогда

$$
S_{p}(\ell) \stackrel{p}{\gtrsim} \begin{cases}\ell^{p}, & 0 \leqslant p \leqslant 1 \\ \ell, & p \geqslant 1\end{cases}
$$

ДокАзАТЕЛьство. Как и выше, нам достаточно проверить, что неравенства выполнены равномерно по $t \in O_{K}$ при замене $S_{p}(\ell)$ на

$$
\int_{S^{1}}|u(x+\ell)-u(x)|^{p} d x
$$

Используя неравенство Гёльдера, можно ограничиться рассмотрением случая $p \geqslant 1$. Мы опять предполагаем до конца доказательства, что $t \in O_{K}$. 
Пусть $z$ такое же, как в доказательстве леммы 4.15. Тогда

$$
\begin{aligned}
\int_{S^{1}}|u(x+\ell)-u(x)|^{p} d x & \\
\geqslant & \int_{z-\ell / 2}^{z}|\underbrace{\int_{x}^{x+\ell} u^{\prime-}(y) d y}_{\text {обрывы }}-\underbrace{\int_{x}^{x+\ell} u^{\prime+}(y) d y}_{\text {пандусы }}|^{p} d x .
\end{aligned}
$$

Так как $\ell \geqslant C_{1} \nu=K^{-2} \nu / 4$, то, используя (53) при $x \in[z-\ell / 2, z]$, имеем

$$
\int_{x}^{x+\ell} u^{\prime-}(y) d y \geqslant \int_{z}^{z+K^{-2} \nu / 8} u^{\prime-}(y) d y \geqslant \frac{1}{16} K^{-3} .
$$

С другой стороны, так как $\ell \leqslant C_{2}$, то из (46) и (51) следует, что

$$
\int_{x}^{x+\ell} u^{\prime+}(y) d y \leqslant C_{2} K=\frac{1}{20} K^{-3} .
$$

Таким образом,

$$
\int_{S^{1}}|u(x+\ell)-u(x)|^{p} d x \geqslant \frac{1}{2} \ell\left(\left(\frac{1}{16}-\frac{1}{20}\right) K^{-3}\right)^{p} \geqslant C(p) \ell .
$$

Лемма доказана.

Комбинируя полученные выше результаты, мы получаем следующую теорему.

Tеорема 4.17. Пусть $\ell \in J_{1}$. Тогда

$$
S_{p}(\ell) \stackrel{p}{\sim} \begin{cases}\ell^{p}, & 0 \leqslant p \leqslant 1 \\ \ell^{p} \nu^{-(p-1)}, & p \geqslant 1 .\end{cases}
$$

Eсли $\ell \in J_{2}$, mo

$$
S_{p}(\ell) \stackrel{p}{\sim} \begin{cases}\ell^{p}, & 0 \leqslant p \leqslant 1 \\ \ell, & p \geqslant 1 .\end{cases}
$$

Следующий результат непосредственно следует из определения (43).

СлеДСтвиЕ 4.18. Пусть $\ell \in J_{2}$. Тогда эксцесс $F(\ell)$ допускает ощенку $F(\ell) \sim \ell^{-1}$.

По теореме 4.8 при $m \geqslant 1$ мы имеем

$$
\left\{|\widehat{u}(k)|^{2}\right\} \leqslant(2 \pi k)^{-2 m}\left\{\|u\|_{m}^{2}\right\} \stackrel{m}{\sim}(k \nu)^{-2 m} \nu .
$$

Отсюда следует, что $\left\{|\widehat{u}(k)|^{2}\right\}$ при $|k| \succeq \nu^{-1}$ убывает быстрее чем полиномиально.

Теперь займемся оценками $u$ в нормах пространства $H^{s}$ при $s \in(0,1)$.

Лемма 4.19. Имеет место оценка

$$
\left\{\|u\|_{1 / 2}^{2}\right\} \sim|\log \nu| .
$$


ДокАЗАтЕЛЬСтво. Из (5) имеем

$$
\|u\|_{1 / 2} \sim\left(\int_{S^{1}}\left(\int_{0}^{1} \frac{|u(x+\ell)-u(x)|^{2}}{\ell^{2}} d \ell\right) d x\right)^{1 / 2} .
$$

Следовательно, по теореме Фубини

$$
\begin{aligned}
\left\{\|u\|_{1 / 2}^{2}\right\} & \sim \int_{0}^{1} \frac{1}{\ell^{2}}\left\{\int_{S^{1}}|u(x+\ell)-u(x)|^{2} d x\right\} d \ell \\
& =\int_{0}^{1} \frac{S_{2}(\ell)}{\ell^{2}} d \ell=\int_{J_{1}} \frac{S_{2}(\ell)}{\ell^{2}} d \ell+\int_{J_{2}} \frac{S_{2}(\ell)}{\ell^{2}} d \ell+\int_{J_{3}} \frac{S_{2}(\ell)}{\ell^{2}} d \ell .
\end{aligned}
$$

Далее, используя теорему 4.17, мы получаем

$$
\int_{J_{1}} \frac{S_{2}(\ell)}{\ell^{2}} d \ell \sim \int_{0}^{C_{1} \nu} \frac{\ell^{2} \nu^{-1}}{\ell^{2}} d \ell \sim 1
$$

и

$$
\int_{J_{2}} \frac{S_{2}(\ell)}{\ell^{2}} d \ell \sim \int_{C_{1} \nu}^{C_{2}} \frac{\ell}{\ell^{2}} d \ell \sim|\log \nu|
$$

Окончательно, из леммы 4.10 имеем

$$
\int_{J_{3}} \frac{S_{2}(\ell)}{\ell^{2}} d \ell \leqslant C C_{2}^{-2} \leqslant C
$$

Таким образом,

$$
\left\{\|u\|_{1 / 2}^{2}\right\} \sim|\log \nu|
$$

Лемма доказана.

Доказательство следующего результата основано на аналогичных рассуждениях.

Лемма 4.20. Пусть $s \in(0,1 / 2)$. Тогда

$$
\left\{\|u\|_{s}^{2}\right\} \stackrel{s}{\sim} 1
$$

Далее, если $s \in(1 / 2,1)$, то

$$
\left\{\|u\|_{s}^{2}\right\} \stackrel{s}{\sim} \nu^{-(2 s-1)}
$$

Из приведенных выше результатов вытекает, что $\left\{|\widehat{u}(k)|^{2}\right\}$ убывает очень быстро при $|k| \gtrsim \nu^{-1}$ и что при $s \geqslant 0$ поведение суммы $\sum|k|^{2 s}\left\{|\widehat{u}(k)|^{2}\right\}$ в точности совпадает с поведением частичных сумм $\sum_{|k| \leqslant \nu^{-1}}|k|^{2 s}|k|^{-2}$ при $\nu \rightarrow 0^{+}$. Как следствие, мы можем предполагать, что $\left\{|\widehat{u}(k)|^{2}\right\} \sim|k|^{-2}$ при $|k| \lesssim \nu^{-1}$.

В действительности результат такого типа имеет место (после усреднения по слоям) при не слишком малых $|k|$. Это утверждение вытекает из того, что

$$
|v(\cdot+y)-v(\cdot)|^{2}=4 \sum_{n \in \mathbb{Z}} \sin ^{2}(\pi n y)|\widehat{v}(n)|^{2},
$$

как следует из теоремы Парсеваля, примененной к функции $v \in L_{2}$. 
Tеорема 4.21. Пустъ $k$ таково, что $k^{-1} \in J_{2}$. Тогда $E(k) \sim k^{-2}$.

ДокАзАТЕльство. Напомним, что по определению (44)

$$
E(k)=\left\{\frac{\sum_{|n| \in\left[M^{-1} k, M k\right]}|\widehat{u}(n)|^{2}}{\sum_{|n| \in\left[M^{-1} k, M k\right]} 1}\right\} .
$$

Таким образом, теорема будет доказана, если мы покажем, что

$$
\sum_{|n| \in\left[M^{-1} k, M k\right]} n^{2}\left\{|\widehat{u}(n)|^{2}\right\} \sim k .
$$

В дальнейшем мы будем явно указывать зависимость от $M$. Оценка сверху в (55) выполняется без усреднения по $n$ из интервала

$$
|n| \in\left[M^{-1} k, M k\right] .
$$

Действительно, из (42) следует, что

$$
\left\{|\widehat{u}(n)|^{2}\right\} \leqslant C n^{-2} .
$$

Из этого неравенства также вытекает, что

$$
\sum_{|n|<M^{-1} k} n^{2}\left\{|\widehat{u}(n)|^{2}\right\} \leqslant C M^{-1} k
$$

и

$$
\sum_{|n|>M k}\left\{|\widehat{u}(n)|^{2}\right\} \leqslant C M^{-1} k^{-1} .
$$

Для доказательства оценки снизу в (55) заметим, что

$$
\begin{aligned}
\sum_{|n| \leqslant M k} n^{2}\left\{|\widehat{u}(n)|^{2}\right\} & \geqslant \frac{k^{2}}{\pi^{2}} \sum_{|n| \leqslant M k} \sin ^{2}\left(\pi n k^{-1}\right)\left\{|\widehat{u}(n)|^{2}\right\} \\
& \geqslant \frac{k^{2}}{\pi^{2}}\left(\sum_{n \in \mathbb{Z}} \sin ^{2}\left(\pi n k^{-1}\right)\left\{|\widehat{u}(n)|^{2}\right\}-\sum_{|n|>M k}\left\{|\widehat{u}(n)|^{2}\right\}\right) .
\end{aligned}
$$

Используя (54) и (57), имеем

$$
\begin{aligned}
\sum_{|n| \leqslant M k} n^{2}\left\{|\widehat{u}(n)|^{2}\right\} & \geqslant \frac{k^{2}}{4 \pi^{2}}\left(\left\{\left|u\left(\cdot+k^{-1}\right)-u(\cdot)\right|^{2}\right\}-C M^{-1} k^{-1}\right) \\
& \geqslant \frac{k^{2}}{4 \pi^{2}}\left(S_{2}\left(k^{-1}\right)-C M^{-1} k^{-1}\right) .
\end{aligned}
$$

Окончательно, по теореме 4.17

$$
\sum_{|n| \leqslant M k} n^{2}\left\{|\widehat{u}(n)|^{2}\right\} \geqslant\left(C-C M^{-1}\right) k .
$$

Теперь (55) следует из (56) при достаточно большом $M \geqslant 1$. Теорема доказана.

2 УМН, т. 69 , вып. 6 


\section{5. Оценки в случае случайного возмущения}

5.1. Вводная часть. Результаты данного раздела (оценки соболевских норм и мелкомасштабных величин) получены в [12] для уравнения с возмущением белым шумом. В более простом случае возмущения толчками оценки соболевских норм содержатся в [11]. Поскольку такие оценки используются в качестве черного ящика при изучении мелкомасштабных величин, то случай возмущения толчками получается непосредственным обобщением мелкомасштабных оценок из [12]. Таким образом, в данном разделе мы будем рассматривать только уравнение (10) с возмущением белым шумом.

Некоторые доказательства из [12] аналогичны рассуждениям при отсутствии возмущения. Мы приведем только доказательства теоремы 5.1 и леммы 5.6, а также дадим несколько комментариев по доказательству мелкомасштабных результатов.

Для простоты в случае возмущения белым шумом мы будем предполагать, что начальное условие $u_{0}$ является детерминированным. Тем не менее, не составляет труда перенести все результаты на случай случайного начального условия $u_{0}$, независимого от $w(t), t \geqslant 0$. Действительно, в этом случае для любого измеримого функционала $\Phi(u(\cdot))$ мы имеем

$$
\mathrm{E} \Phi(u(\cdot))=\int \mathrm{E}\left(\Phi(u(\cdot)) \mid u(0)=u_{0}\right) d \mu\left(u_{0}\right),
$$

где $\mu\left(u_{0}\right)$ - закон распределения начального условия $u_{0}$. Отметим, что все оценки выполнены равномерно по $u_{0}$.

Более того, при $\tau \geqslant 0$, если $u_{0}$ не зависит от $w(t)-w(\tau), t \geqslant \tau$, имеет место следующее марковское свойство, которое можно установить так же, как в [43]:

$$
\mathrm{E} \Phi(u(\cdot))=\int \mathrm{E}\left(\Phi(u(\tau+\cdot)) \mid u(\tau)=u_{0}\right) d \mu\left(u_{0}\right)
$$

Как следствие, все оценки, имеющие место для времени $t$ или временного интервала $[t, t+T]$, в действительности имеют место для времени $t+\tau$ или временного интервала $[t+\tau, t+\tau+T]$ равномерно по $\tau \geqslant 0$.

Эти же замечания верны для уравнения с возмущением толчками с учетом некоторых естественных модификаций из-за того, что в этом случае возмущение дискретно по времени.

5.2. Оценки соболевских норм. Следующая ключевая оценка доказывается с использованием стохастического варианта принципа максимума Кружкова [40].

Теорема 5.1. Рассмотрим случайную величину

$$
X_{t}=\max _{s \in[t, t+1], x \in S^{1}} u_{x}(s, x) .
$$

Тогда для любого $k \geqslant 1$

$$
\mathrm{E} X_{t}^{k} \stackrel{k}{\lesssim} 1, \quad t \geqslant 1
$$


ДоказАтельство. Положим $t=1$ и обозначим $X_{t}$ через $X$.

Рассмотрим уравнение (10) на временном интервале [0,2]. Полагая $v=u-w$ и дифференцируя один раз в пространстве, мы получаем

$$
\frac{\partial v_{x}}{\partial t}+f^{\prime \prime}(u)\left(v_{x}+w_{x}\right)^{2}+f^{\prime}(u)\left(v_{x}+w_{x}\right)_{x}=\nu\left(v_{x}+w_{x}\right)_{x x}
$$

Далее, положим $\widetilde{v}(t, x)=t v_{x}(t, x)$ и умножим (58) на $t^{2}$. При $t>0$ для $\widetilde{v}$ имеет место уравнение

$$
t \widetilde{v}_{t}-\widetilde{v}+f^{\prime \prime}(u)\left(\widetilde{v}+t w_{x}\right)^{2}+t f^{\prime}(u) \widetilde{v}_{x}+t^{2} f^{\prime}(u) w_{x x}=\nu t \widetilde{v}_{x x}+\nu t^{2} w_{x x x} .
$$

Теперь отметим, что если функция с нулевым средним $\widetilde{v}$ не равна тождественно нулю на области $S=[0,2] \times S^{1}$, то она достигает своего (положительного) максимума $N$ на $S$ в точке $\left(t_{1}, x_{1}\right)$, при этом $t_{1}>0$. В точке $\left(t_{1}, x_{1}\right)$ мы имеем $\widetilde{v}_{t} \geqslant 0, \widetilde{v}_{x}=0$ и $\widetilde{v}_{x x} \leqslant 0$. Далее, в силу $(59)$ в точке $\left(t_{1}, x_{1}\right)$ выполнено неравенство

$$
f^{\prime \prime}(u)\left(\widetilde{v}+t w_{x}\right)^{2} \leqslant \widetilde{v}-t^{2} f^{\prime}(u) w_{x x}+\nu t^{2} w_{x x x} .
$$

Рассмотрим случайную величину

$$
A=\max _{t \in[0,2]}|w(t)|_{3, \infty}
$$

Поскольку $t v(t)$ является первообразной с нулевым средним от $\widetilde{v}(t)$ на $S^{1}$ для каждого $t$, то

$$
\max _{t \in[0,2], x \in S^{1}}|t u| \leqslant \max _{t \in[0,2], x \in S^{1}}(|t v|+|t w|) \leqslant N+2 \max _{t \in[0,2]}|w(t)|_{\infty} \leqslant N+2 A .
$$

Введем величину

$$
\delta=2-h(1)
$$

(cp. с (13)). Так как $\delta>0$, то

$$
\begin{aligned}
\max _{t \in[0,2], x \in S^{1}}\left|t^{2} f^{\prime}(u) w_{x x}\right| & \leqslant A \max _{t \in[0,2], x \in S^{1}} t^{\delta}\left|t^{2-\delta} f^{\prime}(u)\right| \\
& \leqslant C A \max _{t \in[0,2], x \in S^{1}} t^{\delta}(|t u|+t)^{2-\delta} \\
& \leqslant C A(N+2 A+2)^{2-\delta}
\end{aligned}
$$

С этого момента мы будем предполагать, что $N \geqslant 2 A$. Так как $\nu \in(0,1]$ и $f^{\prime \prime} \geqslant \sigma$, то из $(60)$ вытекает, что

$$
\sigma(N-2 A)^{2} \leqslant N+C A(N+2 A+2)^{2-\delta}+4 A .
$$

Таким образом, нами установлено, что если $N \geqslant 2 A$, то $N \leqslant C(A+1)^{1 / \delta}$. Поскольку по (9) все моменты случайной величины $A$ конечны, то моменты $N$ также конечны. По определению $\widetilde{v}$ и $S$ это также верно для $X$. Это доказывает теорему. 
СлеДСТВИЕ 5.2. Пусть $k \geqslant 1$. Тогда

$$
\mathrm{E} \max _{s \in[t, t+1]}|u(s)|_{1,1}^{k} \stackrel{k}{\lesssim} 1, \quad t \geqslant 1 .
$$

СлЕДСТВиЕ 5.3. Пусть $k \geqslant 1$. Тогда

$$
\mathrm{E} \max _{s \in[t, t+1]}|u(s)|_{p}^{k} \stackrel{k}{\lesssim} 1, \quad p \in[1, \infty], \quad t \geqslant 1 .
$$

Лемма 5.4. Пусть $m \geqslant 1$. Тогда

$$
\mathrm{E} \max _{s \in[t, t+1]}\|u(s)\|_{m}^{2} \stackrel{m}{\lesssim} \nu^{-(2 m-1)}, \quad t \geqslant 2 .
$$

Tеорема 5.5. Пусть $m \in\{0,1\}, p \in[1, \infty]$ или $m \geqslant 2, p \in(1, \infty]$. Тогда

$$
\left(\mathrm{E} \max _{s \in[t, t+1]}|u(s)|_{m, p}^{\alpha}\right)^{1 / \alpha} \stackrel{m, p, \alpha}{\lesssim} \nu^{-\gamma}, \quad \alpha>0, \quad t \geqslant 2 .
$$

Теперь сформулируем ключевую оценку снизу.

Лемма 5.6. Существует константа $T_{0}>0$ такая, что

$$
\left(\frac{1}{T} \int_{t}^{t+T} \mathrm{E}\|u(s)\|_{1}^{2} d s\right)^{1 / 2} \gtrsim \nu^{-1 / 2}, \quad t \geqslant 1, \quad T \geqslant T_{0} .
$$

ДокАЗАтЕльСтво. Пусть $T>0$. Тогда, в силу (12)

$$
\mathrm{E}|u(t+T)|^{2} \geqslant \mathrm{E}\left(|u(t+T)|^{2}-|u(t)|^{2}\right)=T I_{0}-2 \nu \int_{t}^{t+T} \mathrm{E}\|u(s)\|_{1}^{2} d s .
$$

С другой стороны, по следствию 5.3 существует константа $C^{\prime}>0$ такая, что $\mathrm{E}|u(t+T)|^{2} \leqslant C^{\prime}$. Следовательно, при $T \geqslant T_{0}:=\left(C^{\prime}+1\right) / I_{0}$ имеем

$$
\frac{1}{T} \int_{t}^{t+T} \mathrm{E}\|u(s)\|_{1}^{2} d s \geqslant \frac{T I_{0}-C^{\prime}}{2 T} \nu^{-1} \geqslant \frac{I_{0}}{2\left(C^{\prime}+1\right)} \nu^{-1},
$$

что доказывает лемму.

Tеорема 5.7. Пусть $m \in\{0,1\}, p \in[1, \infty]$ или $m \geqslant 2, p \in(1, \infty]$. Тогда

$$
\left(\frac{1}{T} \int_{t}^{t+T} \mathrm{E}|u(s)|_{m, p}^{\alpha} d s\right)^{1 / \alpha} \stackrel{m, p, \alpha}{\sim} \nu^{-\gamma}, \quad \alpha>0, \quad t \geqslant T_{1}=T_{0}+2, \quad T \geqslant T_{0} .
$$

Более того, оценки сверху такюе вернъ при замене усреднения по времени максимизачией по $[t, t+1]$, m.e.

$$
\left(\mathrm{E} \max _{s \in[t, t+1]}|u(s)|_{m, p}^{\alpha}\right)^{1 / \alpha} \stackrel{m, p, \alpha}{\lesssim} \nu^{-\gamma}, \quad \alpha>0, \quad t \geqslant 2 .
$$


Нижние оценки верны при всех $m \geqslant 0$ и $p \in[1, \infty]$. Асимптотика (61) имеет место без усреднения по времени, если $m$ и р удовлетворяют равенству $\gamma(m, p)=0$. В таком случае

$$
\left(\mathrm{E}|u(t)|_{m, p}^{\alpha}\right)^{1 / \alpha} \underset{m, p, \alpha}{\sim} 1, \quad \alpha>0, \quad t \geqslant T_{1} .
$$

5.3. Оценки мелкомасштабных величин. Пусть $A$ - наблюдаемая, т. е. действительный функционал на пространстве Соболева $H^{m}$. Мы оценим $A$ на решениях $u^{\omega}(s)$. Через $\{A\}$ обозначим среднее $A\left(u^{\omega}(s)\right)$ по ансамблю и времени на $\left[t, t+T_{0}\right]$ :

$$
\{A\}=\frac{1}{T_{0}} \int_{t}^{t+T_{0}} \mathrm{E} A\left(u^{\omega}(s)\right) d s, \quad t \geqslant T_{1} .
$$

Здесь константа $T_{1}$ та же, что в теореме 5.7.

В данном пункте мы предполагаем, что $\nu \leqslant \nu_{0}$, где $\nu_{0}-$ положительная константа. В этой ситуации определения и выбор $\nu_{0}$, интервалов $J_{1}, J_{2}, J_{3}$ и мелкомасштабных величин - буквально те же (с точностью до изменения смысла скобок $\{\cdot\})$, что в случае отсутствия возмущения.

Лемма 5.8. Пусть $\alpha \geqslant 0 u \ell \in[0,1]$. Тогда

$$
S_{p, \alpha}(\ell) \stackrel{p, \alpha}{\lesssim} \begin{cases}\ell^{\alpha p}, & 0 \leqslant p \leqslant 1 \\ \ell^{\alpha p} \nu^{-\alpha(p-1)}, & p \geqslant 1 .\end{cases}
$$

Лемма 5.9. Пусть $\alpha \geqslant 0 u \ell \in J_{2} \cup J_{3}$. Тогда

$$
S_{p, \alpha}(\ell) \stackrel{p, \alpha}{\lesssim} \begin{cases}\ell^{\alpha p}, & 0 \leqslant p \leqslant 1 \\ \ell^{\alpha}, & p \geqslant 1 .\end{cases}
$$

В следующей лемме утверждается, что с не слишком малой вероятностью и в течение не слишком малого временного интервала ряд соболевских норм имеют тот же порядок, что и их математические ожидания.

ОПРЕДЕЛЕНИЕ 5.10. Для данного решения $u(s)=u^{\omega}(s)$ и $K>1$ мы обозначим через $L_{K}$ множество всех $(s, \omega) \in\left[t, t+T_{0}\right] \times \Omega$ таких, что

$$
\begin{gathered}
K^{-1} \leqslant|u(s)|_{\infty} \leqslant \max u_{x}(s) \leqslant K, \\
K^{-1} \nu^{-1} \leqslant|u(s)|_{1, \infty} \leqslant K \nu^{-1} \\
|u(s)|_{2, \infty} \leqslant K \nu^{-2} .
\end{gathered}
$$

Лемма 5.11. Существуют константы $\widetilde{\widetilde{C}}, K_{1}>0$ такие, что при всех $K \geqslant K_{1}$ выполнено неравенство $\rho\left(L_{K}\right) \geqslant \widetilde{C}$. Здесъ $\rho-$ произведение мерь Лебега и вероятностной меры $\mathrm{P} н а\left[t, t+T_{0}\right] \times \Omega$.

ДокАзАТЕльство аналогично рассуждениям в детерминированном случае. Первое отличие состоит в том, что теперь мы рассматриваем произведение меры Лебега и вероятностной меры, а не просто меру Лебега. Другое отличие заключается в том, что теперь оценка сверху выполняется с вероятностью, стремящейся к 1 при $K \rightarrow+\infty$, а не с вероятностью 1 при достаточно больших $K$. 
ОПРЕДЕЛЕНИЕ 5.12. Для заданного решения $u(s)=u^{\omega}(s)$ и $K>1$ мы обозначим через $O_{K}$ множество всех $(s, \omega) \in\left[t, t+T_{0}\right] \times \Omega$, для которых выполнены условия (64), (66) и

$$
K^{-1} \nu^{-1} \leqslant-\min u_{x} \leqslant K \nu^{-1} .
$$

СлеДСтвиЕ 5.13. Пусть $K \geqslant K_{1}, \nu<K_{1}^{-2}$. Тогда $\rho\left(O_{K}\right) \geqslant \widetilde{C}$. Здесъ константы $\widetilde{C}$ и $K_{1}$ такие же, как в утверждении леммы 5.11.

TeOPEma 5.14. Пусть $\alpha \geqslant 0 u \ell \in J_{1}$. Тогда

$$
S_{p, \alpha}(\ell) \stackrel{p, \alpha}{\sim} \begin{cases}\ell^{\alpha p}, & 0 \leqslant p \leqslant 1 \\ \ell^{\alpha p} \nu^{-\alpha(p-1)}, & p \geqslant 1 .\end{cases}
$$

Если $\alpha \geqslant 0 u \ell \in J_{2}$, mo

$$
S_{p, \alpha}(\ell) \stackrel{p, \alpha}{\sim} \begin{cases}\ell^{\alpha p}, & 0 \leqslant p \leqslant 1 \\ \ell^{\alpha}, & p \geqslant 1\end{cases}
$$

СлеДСТвиЕ 5.15. Пусть $\ell \in J_{2}$. Тогда эксцесс $F(\ell)$ допускает оценку $F(\ell) \sim \ell^{-1}$.

ЛЕмма 5.16. Имеют место следующие утверждения:

$$
\begin{array}{rlrl}
\left\{\|u\|_{s}^{2}\right\} & \stackrel{s}{\sim} 1, & s \in\left(0, \frac{1}{2}\right), \\
\left\{\|u\|_{1 / 2}^{2}\right\} & \sim|\log \nu|, & \\
\left\{\|u\|_{s}^{2}\right\} & \stackrel{s}{\sim} \nu^{-(2 s-1)}, \quad s \in\left(\frac{1}{2}, 1\right) .
\end{array}
$$

Теорема 5.17. Если $M$ в определении $E(k)$ достаточно велико, то $E(k) \sim$ $k^{-2}$ для каждого $k$ при условии, что $k^{-1} \in J_{2}$. Более того,

$$
\left\{\left(\frac{\sum_{|n| \in\left[M^{-1} k, M k\right]}|\widehat{u}(n)|^{2}}{\sum_{|n| \in\left[M^{-1} k, M k\right]} 1}\right)^{\alpha}\right\} \stackrel{\alpha}{\sim} k^{-2 \alpha}, \quad \alpha>0 .
$$

\section{6. Стационарная мера и сопутствующие вопросы}

Результаты данного раздела получены автором в [12] для уравнения с белым шумом. С точностью до некоторых естественных модификаций, необходимых из-за того, что возмущение дискретно по времени, данные результаты могут быть обобщены на случай возмущения толчками. По поводу деталей мы отсылаем к работе [10]; см. также [43], где вводится случайное возмущение в аналогичной ситуации.

ТеОРема 6.1. Пусть и, $\bar{u}-$ два решения уравнения (10), соответствующие одной и той же случайной силе, но с разными начальными условиями из $C^{\infty}$. Тогда при всех $t \geqslant 0$

$$
|u(t)-\bar{u}(t)|_{1} \leqslant|u(0)-\bar{u}(0)|_{1} .
$$


Так как пространство $C^{\infty}$ плотно в $L_{1}$, то теорема 6.1 позволяет нам обобщить определение потока, соответствующего уравнению (10), рассматривая теперь начальное условие из $L_{1}$. Отметим, что поскольку поток Бюргерса является сглаживающим, то соответствующие $L_{1}$-решения становятся решениями уравнения (10) при $t>0$. Это позволяет нам доказать, что поток, соответствующий (10), индуцирует непрерывный по времени марковский процесс, так что мы можем определить соответствующую полугруппу $S_{t}^{*}$, действующую на борелевских мерах на $L_{1}$. Вопросы корректности постановки в подобной ситуации подробно разобраны в монографии [43].

Стационарная мера - это борелевская вероятностная мера на $L_{1}$, являющаяся инвариантной относительно полугруппы $S_{t}^{*}$ для каждого $t$. Cmauионарное решение уравнения (10) - это случайный процесс $v$, определенный при $(t, \omega) \in[0,+\infty) \times \Omega$, принимающий значения из $L_{1}$, такой, что распределение $v(t)$ не зависит от $t$, и удовлетворяющий (10). Такое распределение автоматически является стационарной мерой.

Рассмотрим теперь вопрос существования и единственности стационарной меры, что влечет существование и единственность (с точностью до распределения) стационарного решения. Кроме того, мы рассмотрим скорость сходимости к стационарной мере. Полученная оценка не зависит от вязкости или начальных условий.

ОПРЕДЕЛЕНИЕ 6.2. Пусть $p \in[1, \infty)$. Под липиищевой нормой непрерывной функции

$$
g: L_{p} \rightarrow \mathbb{R}
$$

мы понимаем величину

$$
|g|_{L}:=\sup _{L_{p}}|g|+|g|_{\text {Lip }}
$$

где $|g|_{\text {Lip }}$ - липшицева константа функции $g$. Через $L(p)=L\left(L_{p}\right)$ мы обозначаем класс непрерывных функций с конечной липшицевой нормой. Для краткости мы будем писать $L$ вместо $L(1)$.

ОПРЕДЕЛЕНИЕ 6.3. Для борелевских вероятностных мер $\mu_{1}, \mu_{2}$ на $L_{p}$ мы обозначим через $\left\|\mu_{1}-\mu_{2}\right\|_{L(p)}^{*}$ двойственное липшицево расстояние:

$$
\left\|\mu_{1}-\mu_{2}\right\|_{L(p)}^{*}:=\sup _{g \in L(p),|g|_{L(p)} \leqslant 1}\left|\int_{S^{1}} g d \mu_{1}-\int_{S^{1}} g d \mu_{2}\right| .
$$

Имея в нашем распоряжении $u_{0}$-равномерные оценки сверху, мы устанавливаем существование стационарной меры для обобщенного уравнения Бюргерса с помощью аргументов Боголюбова-Крылова (см. [43]).

Теперь мы можем сформулировать основной результат данного раздела, из которого сразу вытекает единственность стационарной меры $\mu$ для уравнения (10), а также оценка скорости сходимости к данной мере, являющаяся алгебраической по $t$ и равномерной по коэффициенту вязкости $\nu$. Теорема 6.4 доказывается с использованием упрощенного варианта метода каплинга, предложенного С.Б. Куксиным и А.Р. Ширикяном [43; гл. 3]. Отметим, 
что здесь ситуация более простая, чем для стохастического двумерного уравнения Навье-Стокса. Действительно, в нашей ситуации "время затухания", необходимое для того, чтобы сделать малым расстояние между двумя решениями, соответствующими одному и тому же возмущению, не зависит от начальных условий и, более того, по теореме 6.1 поток уравнения (10) является $L_{1}$-сжимающим.

Теорема 6.4. Существует положительная константа $C^{\prime}$ такая, что

$$
\left\|S_{t}^{*} \mu_{1}-S_{t}^{*} \mu_{2}\right\|_{L}^{*} \leqslant C^{\prime} t^{-1 / 13}, \quad t \geqslant 1
$$

для любах вероятностных мер $\mu_{1}, \mu_{2}$ на $L_{1}$.

СлеДСтвиЕ 6.5. Для каждого $p \in(1, \infty)$ существует положительная константа $C^{\prime}(p)$ такая, что

$$
\left\|S_{t}^{*} \mu_{1}-S_{t}^{*} \mu_{2}\right\|_{L(p)}^{*} \leqslant C^{\prime} t^{-1 /(13 p)}, \quad t \geqslant 1,
$$

для любых вероятностных мер $\mu_{1}, \mu_{2}$ на $L_{p}$.

Отметим, что все оценки из предыдущих разделов по-прежнему верны для стационарного решения, поскольку они выполнены равномерно при любых начальных данных из $L_{1}$ для большого времени, а статистические свойства стационарного решения не зависят от времени. Отсюда следует, что данные оценки также верны при замене усреднения по времени и по ансамблю (что обозначается как $\{\cdot\}$ ) на усреднение только по ансамблю, т. е. при усреднении по $\mu$. Именно, из теорем 5.7, 5.14 и 5.17 вытекают соответственно следующие результаты.

Теорема 6.6. Пусть $m \in\{0,1\}, p \in[1, \infty]$ или $m \geqslant 2, p \in(1, \infty]$. Тогда

$$
\left(\int|u|_{m, p}^{\alpha} d \mu(u)\right)^{1 / \alpha} \stackrel{m, p, \alpha}{\sim} \nu^{-\gamma}, \quad \alpha>0 .
$$

Tеорема 6.7. Пусть $\alpha \geqslant 0, \ell \in J_{1}$. Тогда

$$
\int\left(\int_{S^{1}}|u(x+\ell)-u(x)|^{p} d x\right)^{\alpha} d \mu(u) \stackrel{p, \alpha}{\sim} \begin{cases}\ell^{\alpha p}, & 0 \leqslant p \leqslant 1 \\ \ell^{\alpha p} \nu^{-\alpha(p-1)}, & p \geqslant 1\end{cases}
$$

Если $\alpha \geqslant 0$ u $\ell \in J_{2}$, mo

$$
\int\left(\int_{S^{1}}|u(x+\ell)-u(x)|^{p} d x\right)^{\alpha} d \mu(u) \stackrel{p, \alpha}{\sim} \begin{cases}\ell^{\alpha p}, & 0 \leqslant p \leqslant 1 \\ \ell^{\alpha}, & p \geqslant 1 .\end{cases}
$$

Теорема 6.8. Пусть $k$ таково, что $k^{-1} \in J_{2}$. Тогда

$$
\int \frac{\sum_{|n| \in\left[M^{-1} k, M k\right]}|\widehat{u}(n)|^{2}}{\sum_{|n| \in\left[M^{-1} k, M k\right]} 1} d \mu(u) \sim k^{-2} .
$$


Я очень благодарен Ю. Ю. Бахтину, А. Э. Бирюку, К. М. Ханину, С. Б. Куксину, У. Фришу (U. Frisch) и А.Р. Ширикяну за полезные обсуждения. Часть настоящей работы была написана во время моей работы на факультете математики университета Сержи-Понтуаза и на факультете физики университета Женевы при поддержке соответственно грантами ERC BLOWDISOL и ERC BRIDGES. Я также выражаю благодарность коллективам данных университетов и, в особенности, Ф. Мерлю (F. Merle) и Ж.-П. Экманну (J.-P. Eckmann) за гостеприимство.

\section{Список литературы}

[1] R. A. Adams, Sobolev spaces, Pure Appl. Math., 65, Academic Press, New YorkLondon, 1975, xviii+268 pp.

[2] E. Aurell, U. Frisch, J. Lutsko, M. Vergassola, "On the multifractal properties of the energy dissipation derived from turbulence data", J. Fluid Mech., 238 (1992), 467-486.

[3] Y. Bakhtin, "Ergodic theory of the Burgers equation with random force", St. Petersburg summer school in probability and statistical physics (St. Petersburg, 2012), eds. V. Sidoravicius, S. Smirnov (to appear).

[4] G. K. Batchelor, The theory of homogeneous turbulence, Cambridge Monogr. Mech. Appl. Math., Cambridge Univ. Press, Cambridge, 1953, x+197 pp.

[5] H. Bateman, "Some recent researches on the motion of fluids", Mon. Wea. Rev., 1915, № 43, 163-170.

[6] J. Bec, K. Khanin, "Burgers turbulence", Phys. Rep., 447:1-2 (2007), 1-66.

[7] А. Э. Бирюк, "Спектральные свойства решений уравнения Бюргерса с малой диссипацией”, Функи. анализ и его прил., 35:1 (2001), 1-15; англ. пер.: А. Ѐ. Biryuk, "Spectral properties of solutions of the Burgers equation with small dissipation", Funct. Anal. Appl., 35:1 (2001), 1-12.

[8] A. Biryuk, Note on the transformation that reduces the Burgers equation to the heat equation, Mathematical Physics Preprint Archive, MP-ARC-2003-370, 2003, 4 pp.

[9] A. Boritchev, "Decaying turbulence in the generalised Burgers equation", Arch. Ration. Mech. Anal., 214:1 (2014), 331-357; 2012 (v4-2014), 28 pp., arXiv: 1208.5241.

[10] A. Boritchev, Generalised Burgers equation with random force and small viscosity, $\mathrm{PhD}$ thesis, École Polytechnique, 2012, 156 pp.

[11] A. Boritchev, "Estimates for solutions of a low-viscosity kick-forced generalised Burgers equation", Proc. Roy. Soc. Edinburgh Sect. A, 143:2 (2013), 253-268.

[12] A. Boritchev, "Sharp estimates for turbulence in white-forced generalised Burgers equation", Geom. Funct. Anal., 23:6 (2013), 1730-1771.

[13] J. M. Burgers, "A mathematical model illustrating the theory of turbulence", Adv. Appl. Mech., 1, Academic Press, Inc., New York, NY, 1948, 171-199.

[14] J. M. Burgers, The nonlinear diffusion equation. Asymptotic solutions and statistical problems, Reidel, Dordrecht-Boston, 1974, x+173 pp.

[15] A. J. Chorin, Lectures on turbulence theory, Math. Lecture Ser., 5, Publish or Perish, Inc., Boston, MA, 1975, iii+159 pp.

[16] J.D. Cole, "On a quasilinear parabolic equation occurring in aerodynamics", Quart. Appl. Math., 9 (1951), 225-236. 
[17] G. Da Prato, J. Zabczyk, Stochastic equations in infinite dimensions, Encyclopedia Math. Appl., 44, Cambridge Univ. Press, Cambridge, 1992, xviii+454 pp.

[18] G. Da Prato, J. Zabczyk, Ergodicity for infinite dimensional systems, London Math. Soc. Lecture Note Ser., 229, Cambridge Univ. Press, Cambridge, 1996, xii+339 pp.

[19] C. M. Dafermos, Hyperbolic conservation laws in continuum physics, 3rd ed., Grundlehren Math. Wiss., 325, Springer-Verlag, Berlin, 2010, xxxvi+708 pp.

[20] C. R. Doering, J. D. Gibbon, Applied analysis of the Navier-Stokes equations, Cambridge Texts Appl. Math., Cambridge Univ. Press, Cambridge, 1995, xiv+217 pp.

[21] W. E, K. Khanin, A. Mazel, Ya. Sinai, "Invariant measures for Burgers equation with stochastic forcing", Ann. of Math. (2), 151:3 (2000), 877-960.

[22] L.C. Evans, Partial differential equations, 2nd ed., Grad. Stud. Math., 19, Amer. Math. Soc., Providence, RI, 2010, xxii+749 pp.

[23] В. А. Флорин, "Некоторые простейшие нелинейные задачи консолидации водонасыщенной земляной среды", Изв. АН СССР. ОТН, 1948, № 9, 1389-1402.

[24] A.R. Forsyth, Theory of differential equations, v. 5, 6: Partial differential equations, Cambridge Univ. Press, London, 1906, xx+478 pp., xiii+596 pp.

[25] J.-D. Fournier, U. Frisch, "L'équation de Burgers déterministe et statistique", J. Méc. Théor. Appl., 2:5 (1983), 699-750.

[26] У. Фриш, Турбулентность. Наследие А.Н. Колмогорова, Фазис, М., 1998, 343 с.; пер. с англ.: U. Frisch, Turbulence. The legacy of A. N. Kolmogorov, Cambridge Univ. Press, Cambridge, 1995, xiv+296 pp.

[27] U. Frisch, J. Bec, "Burgulence", Turbulence: nouveaux aspects/New trends in turbulence (Les Houches, 2000), eds. M. Lesieur, A. Yaglom, F. David, EDP Sci., Les Ulis, 2001, 341-383.

[28] D. Gomes, R. Iturriaga, K. Khanin, P. Padilla, "Viscosity limit of stationary distributions for the random forced Burgers equation", Mosc. Math. J., 5:3 (2005), 613-631.

[29] T. Gotoh, R. H. Kraichnan, "Steady-state Burgers turbulence with large-scale forcing", Phys. Fluids, 10:11 (1998), 2859-2866.

[30] E. Hopf, "The partial differential equation $u_{t}+u u_{x}=\mu u_{x x}$ ", Comm. Pure Appl. Math., 3:3 (1950), 201-230.

[31] R. Iturriaga, K. Khanin, "Burgers turbulence and random Lagrangian systems", Comm. Math. Phys., 232:3 (2003), 377-428.

[32] S. Kida, "Asymptotic properties of Burgers turbulence", J. Fluid Mech., 93:2 (1979), $337-377$.

[33] А.Н. Колмогоров, "Рассеяние энергии при локально-изотропной турбулентности", Докл. АН СССР, 32 (1941), 16-18; англ. пер.: A. N. Kolmogorov, "Dissipation of energy in the locally isotropic turbulence", Proc. Roy. Soc. London Ser. A, 434:1890 (1991), 15-17.

[34] А.Н. Колмогоров, "К вырождению изотропной турбулентности в несжимаемой вязкой жидкости", Докл. АН СССР, 31 (1941), 538-540; англ. пер.: A. N. Kolmogorov, "On the degeneration of isotropic turbulence in an incompressible viscous fluid", Selected works of A. N. Kolmogorov, v. I: Mathematics and mechanics, Math. Appl. (Soviet Ser.), 25, Kluwer Academic Publishers Group, Dordrecht, 1991, 319-323.

[35] А.Н. Колмогоров, “Локальная структура турбулентности в несжимаемой вязкой жидкости при очень больших числах Рейнольдса", Докл. АH CCCP, 30 (1941), 299-303; англ. пер.: А. N. Kolmogorov, "The local structure of turbulence in incompressible viscous fluid for very large Reynolds number", Proc. Roy. Soc. London Ser. A, 434:1890 (1991), 9-13. 
[36] A. N. Kolmogorov, "A refinement of previous hypotheses concerning the local structure of turbulence in a viscous incompressible fluid at high Reynolds number", J. Fluid Mech., 13:1 (1962), 82-85.

[37] R. H. Kraichnan, "Lagrangian-history statistical theory for Burgers' equation", Phys. Fluids, 11:2 (1968), 265-277.

[38] H.-O. Kreiss, "Fourier expansions of the solutions of the Navier-Stokes equations and their exponential decay rate", Analyse mathématique et applications, Gauthier-Villars, Montrouge, 1988, 245-262.

[39] H.-O. Kreiss, J. Lorenz, Initial-boundary value problems and the Navier-Stokes equations, Pure Appl. Math., 136, Academic Press, Inc., Boston, MA, 1989, xii+402 pp.

[40] С. Н. Кружков, “Задача Коши в целом для нелинейных уравнений и некоторых квазилинейных систем первого порядка со многими переменными", Докл. АН CCCP, 155 (1964), 743-746; англ. пер.: S. N. Kruzhkov, "The Cauchy problem in the large for nonlinear equations and for certain quasilinear systems of the first-order with several variables", Soviet Math. Dokl., 5 (1964), 493-496.

[41] S. B. Kuksin, "On turbulence in nonlinear Schrödinger equations", Geom. Funct. Anal., 7:4 (1997), 783-822.

[42] S. B. Kuksin, "Spectral properties of solutions for nonlinear PDEs in the turbulent regime", Geom. Funct. Anal., 9:1 (1999), 141-184.

[43] S. Kuksin, A. Shirikyan, Mathematics of two-dimensional turbulence, Cambridge Tracts in Math., 194, Cambridge Univ. Press, Cambridge, 2012, xvi+320 pp.

[44] H.-H. Kuo, Gaussian measures in Banach spaces, Lecture Notes in Math., 463, Springer-Verlag, Berlin-New York, 1975, vi+224 pp.

[45] P. D. Lax, Hyperbolic partial differential equations, Courant Lect. Notes Math., 14, New York Univ., Courant Inst. Math. Sci., New York, NY; Amer. Math. Soc., Providence, RI, 2006, viii+217 pp.

[46] А. М. Обухов, "О распределении энергии в спектре турбулентного потока”, Докл. AH CCCP, 32:1 (1941), 22-24.

[47] А. М. Обухов, "О распределении энергии в спектре турбулентного потока", Изв. АН СССР. Сер. геогр. и геофиз., 5:4 (1941), 453-466.

[48] G. Parisi, U. Frisch, "Fully developed turbulence and intermittency", Turbulence and predictability in geophysical fluid dynamics and climate dynamics, Proceedings of the International School of Physics "Enrico Fermi", eds. M. Ghil, R. Benzi, G. Parisi, North-Holland, Amsterdam, 1985, 71-88.

[49] D. Serre, Systems of conservation laws, v. 1: Hyperbolicity, entropies, shock waves, Cambridge Univ. Press, Cambridge, 1999, xxii+263 pp.

[50] Z.-S. She, S. A. Orszag, "Physical model of intermittency in turbulence: inertial-range non-Gaussian statistics", Phys. Rev. Lett., 66:13 (1991), 1701-1704.

[51] Ya. G. Sinai, "Two results concerning asymptotic behavior of solutions of the Burgers equation with force", J. Statist. Phys., 64:1-2 (1991), 1-12.

[52] Ya. G. Sinai, "Burgers system driven by a periodic stochastic flow", Itô's stochastic calculus and probability theory, Springer, Tokyo, 1996, 347-353.

[53] E. Tadmor, "Total variation and error estimates for spectral viscosity approximations", Math. Comp., 60:201 (1993), 245-256.

[54] M. E. Taylor, Partial differential equations, v. I: Basic theory, Appl. Math. Sci., 115, Springer-Verlag, New York, 1996, xxiv+563 pp. 
[55] A. Tsinober, An informal conceptual introduction to turbulence, Fluid Mech. Appl., 92, Springer, Dordrecht, 2009, xx+464 pp.

[56] J. von Neumann, Collected works (1949-63), v.6: Theory of games, astrophysics, hydrodynamics and meteorology, Pergamon Press Book, The Macmillan Co., New York, 1963, x+538 pp.

\author{
Александр Александрович Боричев \\ (Alexandre A. Boritchev) \\ Institut Camille Jordan, \\ Université Claude Bernard Lyon 1, \\ Villeurbanne, France \\ E-mail: alexandre.boritchev@gmail.com
}

Поступила в редакцию

25.12 .2013 